\title{
Absence of Heating in a Uniform Fermi Gas Created by Periodic Driving
}

\author{
Constantine Shkedrov, ${ }^{1}$ Meny Menashes, ${ }^{1}$ Gal Ness $\odot,{ }^{1}$ Anastasiya Vainbaum, ${ }^{1}$ Ehud Altman, ${ }^{2,3}$ and Yoav Sagi® ${ }^{1, *}$ \\ ${ }^{1}$ Physics Department and Solid State Institute, Technion-Israel Institute of Technology, Haifa 32000, Israel \\ ${ }^{2}$ Department of Physics, University of California, Berkeley, California 94720, USA \\ ${ }^{3}$ Materials Sciences Division, Lawrence Berkeley National Laboratory, Berkeley, California 94720, USA
}

(Received 22 February 2021; accepted 19 January 2022; published 3 March 2022)

\begin{abstract}
Ultracold atomic gas provides a useful tool to explore many-body physics. One of the recent additions to this experimental toolbox is Floquet engineering, where periodic modulation of the Hamiltonian allows the creation of effective potentials that do not exist otherwise. When subject to external modulations, however, generic interacting many-body systems absorb energy, thus posing a heating problem that may impair the usefulness of this method. For discrete systems with bounded local energy, an exponentially suppressed heating rate with driving frequency has been observed previously, leaving the system in a prethermal state for exceedingly long durations. However, for systems in continuous space, the situation remains unclear. Here, we show that Floquet engineering can be applied to a strongly interacting degenerate Fermi gas held in a flat boxlike potential without inducing excessive heating on experimentally relevant timescales. The driving eliminates the effect of a spin-dependent potential originating from the simultaneous magnetic levitation of two different spin states. We calculate the heating rate and obtain a power-law suppression with the drive frequency. To further test the many-body behavior of the driven gas, we measure both the pair-condensation fraction at unitarity and the contact parameter across the BEC-BCS crossover. At low driving frequencies, the condensate fraction is reduced by the time-dependent force, but at higher frequencies, it revives and attains an even higher value than without driving. Our results are promising for future exploration of exotic many-body phases of a bulk strongly interacting Fermi gas with dynamically engineered Hamiltonians.
\end{abstract}

DOI: 10.1103/PhysRevX.12.011041

Subject Areas: Atomic and Molecular Physics, Quantum Physics, Statistical Physics

\section{INTRODUCTION}

The past two decades have witnessed tremendous advance in studying many-body problems with ultracold atomic gases [1]. The vast majority of works are done with static Hamiltonians. Adding periodic driving can generate effective Hamiltonians with completely different properties than the original one-an approach called Floquet engineering [2-4]. For example, modulation of the barrier between potential wells renormalizes the tunneling rate [5-8] and can drive quantum phase transitions [9]. Floquet engineering can also be used to create artificial gauge fields [10-13] and give rise to new phases which do not exist at equilibrium [14,15].

An inherent problem with externally driven systems is their tendency to heat up. Apart from integrable and

\footnotetext{
*yoavsagi@technion.ac.il
}

Published by the American Physical Society under the terms of the Creative Commons Attribution 4.0 International license. Further distribution of this work must maintain attribution to the author(s) and the published article's title, journal citation, and DOI. many-body localized systems, generic interacting ensembles absorb energy from the external force and eventually reach an "infinite temperature" where all states are equally populated [16-18]. Nonetheless, recent theoretical works suggest that, in discrete lattice systems, the energy absorption rate is generally exponentially small in the drive frequency over the energy of a local excitation [19-24]. These predictions are supported by heating rate measurements done with bosons in a driven optical lattice [25]. The exponential suppression of heating in discrete systems relies on the fact that the energy is locally bounded. However, in continuous systems (e.g., bulk quantum gases), there is no such bound. The pertinent question, in this case, is under what conditions one can obtain "cold" prethermal states exhibiting collective phenomena that are governed by an effective "Floquet-engineered" Hamiltonian.

In this paper, we address this question using a driven ultracold Fermi gas near unitarity, exhibiting high- $T_{c}$ fermionic superfluidity [26]. The driving we apply is intended to create a uniform effective potential across the Fermi gas. Although in situ measurements [27-30] and spatial selection [31-36] can give access to quasihomogeneous observables, it is better to create a uniform gas from the outset. This is essential, for example, to study 
critical properties and avoid spurious phase-separated states [37]. Indeed, in recent years, uniform Bose [38,39] and Fermi $[40,41]$ gases have been created in flat optical traps. These traps are formed by several shaped laser beams that create sharp repelling walls enclosing a dark volume.

A significant challenge is posed by the need to offset the gravitational potential, which leads to a substantial energy change in the trap. One obvious solution is to use a shaped optical potential to counter gravity [42]. But generating such a potential, smooth on a nanokelvin scale, is a formidable task. A simpler approach taken in previous experiments is to use a magnetic field with an appropriate gradient. This works if all particles have approximately the same magnetic dipole moment. However, our ${ }^{40} \mathrm{~K}$ Fermi gas is a mixture of two hyperfine states with different (but not opposite) magnetic moments $\mu_{\uparrow} \neq \mu_{\downarrow}$. An appropriate magnetic-field gradient counters the average gravitational potential while leaving an opposite potential gradient on each of the two species [Fig. 1(a)].

To counter the residual field gradient, we apply an rf field that induces a rapid precession at a Rabi frequency $\Omega$. In the rotating frame of the precessing spins, the static spindependent potential gradient is translated to a periodic perturbation of frequency $\Omega$. The rest of the interacting fermion Hamiltonian, including the flat spin-independent potential, is invariant to spin rotations and, therefore, unchanged in the rotating frame [Fig. 1(b)]. Thus, we achieve an ultracold uniform state of a spin-balanced gas of

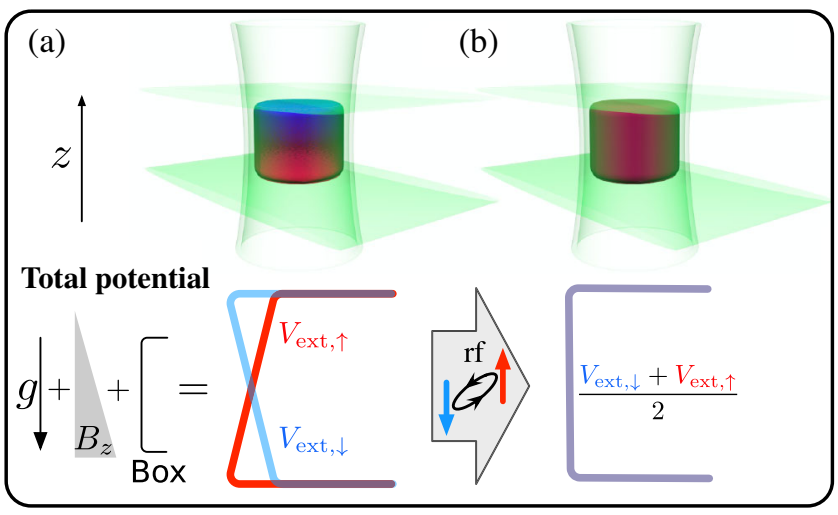

FIG. 1. Creating a uniform Fermi gas by periodic driving. (a) The gas, composed of two spin states (marked by red and blue and by opposite arrows), is trapped in a boxlike optical potential. The two spins have different magnetic dipole moments. As a result, it is possible to only partially counteract the gravitational potential with a magnetic-field set according to Eq. (3). The total external potential $V_{\text {ext, } s}$ depends on the spin $s \in\{\uparrow, \downarrow\}$, and, consequently, the density distribution of each spin is different and not uniform (color gradient in the top left figure). (b) By adding a resonant rf field that drives rapid spin rotations, we create an effective spin-independent potential, in which the gas becomes homogeneous. Importantly, the intrinsic many-body behavior of the gas is unchanged by this driving.
${ }^{40} \mathrm{~K}$ atoms that is useful if the periodic perturbation does not cause significant heating over experimental timescales.

We establish this property by measuring the paircondensate fraction (CF) at unitarity while applying a continuous driving. At low frequencies, driving impairs the gas conditions and reduces the CF. As the frequency increases further, the $\mathrm{CF}$ recovers and even surpasses its value without the driving. At high driving frequencies, we do not detect heating or excessive loss of atoms which can be attributed to the drive. Finally, we perform rf spectroscopy with a uniform gas in the BEC-BCS crossover regime and extract the homogeneous contact parameter as a function of the interaction strength.

The structure of this paper is as follows. In Sec. II, we review the theoretical model for radio frequency driving and calculate the expected heating rate in the presence of this drive. In Sec. III, we describe the experimental setup and measurement sequence. The results are presented in Sec. IV. We study with time-dependent in situ imaging the relaxation dynamics following the application of the driving field. The temperature of the uniform gas is probed by Raman spectroscopy. The many-body behavior of the uniform driven gas is studied with a pair-projection technique and rf spectroscopy. We study both the frequency dependence and the long-time behavior of the driven gas. Section V concludes with a discussion and outlook.

\section{THEORETICAL MODEL}

\section{A. Effective Hamiltonian}

We consider fermions in two possible spin states, denoted by $|\downarrow\rangle$ and $|\uparrow\rangle$, where the energy of the latter is larger by $\hbar \omega_{0}$. The two particles are placed in an external potential and coupled by an rf field with a frequency $\omega_{\mathrm{rf}}$. The Hamiltonian is a sum of three terms $\hat{H}=\hat{H}_{0}+$ $\hat{H}_{\text {int }}+\hat{H}_{\text {rf }}$ that account for the single-particle kinetic and potential energy $\left(\hat{H}_{0}\right)$, the interaction Hamiltonian $\left(\hat{H}_{\text {int }}\right)$, and the coupling to the external rf field $\left(\hat{H}_{\mathrm{rf}}\right)$. In the frame rotating with the $\uparrow$ spin, they are given by $[43,44]$

$\hat{H}_{0}=\sum_{\sigma=\uparrow, \downarrow} \int d^{3} r \hat{\Psi}_{\sigma}^{\dagger}(\mathbf{r})\left(-\frac{\hbar^{2} \nabla^{2}}{2 m}+V_{\sigma}(\mathbf{r})\right) \hat{\Psi}_{\sigma}(\mathbf{r})$,

$\hat{H}_{\mathrm{int}}=\sum_{\sigma \sigma^{\prime}} \iint d^{3} r^{\prime} d^{3} r u\left(\mathbf{r}-\mathbf{r}^{\prime}\right) \hat{\Psi}_{\sigma}^{\dagger}(\mathbf{r}) \hat{\Psi}_{\sigma^{\prime}}^{\dagger}\left(\mathbf{r}^{\prime}\right) \hat{\Psi}_{\sigma^{\prime}}\left(\mathbf{r}^{\prime}\right) \hat{\Psi}_{\sigma}(\mathbf{r})$,

$\hat{H}_{\mathrm{rf}}=\frac{\hbar}{2} \int d^{3} r \Omega e^{i \omega_{0} t}\left(e^{i \omega_{\mathrm{rf}} t}+e^{-i \omega_{\mathrm{rf}} t}\right) \hat{\Psi}_{\uparrow}^{\dagger}(\mathbf{r}) \hat{\Psi}_{\downarrow}(\mathbf{r})+$ H.c.,

where $\Omega$ is the Rabi frequency of the $\operatorname{rf}$ field, $V_{\sigma}(\mathbf{r})$ is the external potential for spin $\sigma$, and $\hat{\Psi}_{\sigma}(\mathbf{r})$ are fermionic 
field operators obeying the anticommutation relation $\left\{\hat{\Psi}_{\sigma}(\mathbf{r}), \hat{\Psi}_{\sigma^{\prime}}^{\dagger}\left(\mathbf{r}^{\prime}\right)\right\}=\delta_{\sigma \sigma^{\prime}} \delta\left(\mathbf{r}-\mathbf{r}^{\prime}\right)$. Note that here we consider a general spin-symmetric (and translationally invariant) two-body interaction. The interaction potential $u\left(r-r^{\prime}\right)$ represents the microscopic interaction rather than a low-energy limit of the $T$ matrix (pseudopotential). In particular, we are interested in a unitary gas for which the $T$ matrix is explicitly energy dependent in the lowenergy limit.

In our experiment, the rf field is resonant with the bare energy difference $\omega_{\mathrm{rf}}=\omega_{0}$, and $\omega_{0} \gg \Omega$. Thus, within the rotating wave approximation, the rf field is seen as a large magnetic field along $S^{x}: \hat{H}_{\mathrm{rf}}=$ $(\hbar / 2) \int d^{3} r \Omega \hat{\Psi}_{\uparrow}^{\dagger}(\mathbf{r}) \hat{\Psi}_{\downarrow}(\mathbf{r})+$ H.c. The initial state is presumed to be an ultracold Fermi gas with equally populated spin components $N_{\uparrow}=N_{\downarrow}$, which in the presence of the field precesses around the $x$ axis at the Rabi frequency $\Omega$. To clearly see the effect of the external rf field, we eliminate the field by a unitary transformation $\hat{U}=e^{(i / \hbar) \hat{H}_{\mathrm{rf}} t}$ into a reference frame that rotates with the spins.

The Hamiltonian transforms in a simple way under $\hat{U}$. The kinetic energy and interaction Hamiltonians are both invariant under spin rotations and are, therefore, unchanged by the time-dependent transformation. As first noted by Zwierlein et al. [45], the invariance of contact interactions under $\mathrm{rf}$ rotations is the reason for the absence of a spectroscopic shift in the transition frequency between the spins. The external potential, on the other hand, can be decomposed into spin-symmetric and -antisymmetric parts:

$$
\sum_{\sigma=\uparrow, \downarrow} V_{\sigma}(\mathbf{r}) \hat{n}_{\sigma}(\mathbf{r})=V(\mathbf{r}) \hat{n}(\mathbf{r})-h(\mathbf{r}) \hat{S}^{z}(\mathbf{r}),
$$

where $\hat{n}(\mathbf{r})=\hat{n}_{\uparrow}(\mathbf{r})+\hat{n}_{\downarrow}(\mathbf{r})$ is the number density, $\hat{S}^{z}(\mathbf{r})=\left[\hat{n}_{\uparrow}(\mathbf{r})-\hat{n}_{\downarrow}(\mathbf{r})\right] / 2$ the spin density, $V(\mathbf{r})=$ $\left[V_{\uparrow}(\mathbf{r})+V_{\downarrow}(\mathbf{r})\right] / 2$, and $h(\mathbf{r})=V_{\downarrow}(\mathbf{r})-V_{\uparrow}(\mathbf{r})$. Only the spin-symmetric part of the potential, which couples to the number density, is invariant under the transformation $\hat{U}$ and does not change with time.

In our experiment, the external potential is given by $V_{\sigma}(\mathbf{r})=V_{\text {trap }}(\mathbf{r})+m g z-\mu_{\sigma} B^{\prime} z$, where the first term is the flat optical potential, the second term is the gravitational potential, and the last term describes the interaction of a spin with a magnetic moment $\mu_{\sigma}$ with the external magnetic field, which is linear in height $z$. Thus, the spinsymmetric part of the potential is $V(\mathbf{r})=V_{\text {trap }}(\mathbf{r})+m g z-$ $B^{\prime} z\left(\mu_{\uparrow}+\mu_{\downarrow}\right) / 2$. By tuning the magnetic-field gradient to the value

$$
B^{\prime}=\frac{2 m g}{\mu_{\uparrow}+\mu_{\downarrow}},
$$

we obtain a flat total potential. The gravitational potential is, thus, eliminated by the static part of the Hamiltonian.
The spin-antisymmetric part of the external potential, which couples to the spin density, gives rise to a timedependent coupling in the rotating frame $\hat{U}$. Specifically, the spin density rotates as $\hat{U} \hat{S}^{z} \hat{U}^{\dagger}=\cos (\Omega t) \hat{S}^{z}-\sin (\Omega t) \hat{S}^{y}$, leading to a Zeeman field rotating at frequency $\Omega$ in the $\hat{S}^{z}, \hat{S}^{y}$ plane. With a simple redefinition of the spin axes, we write the residual time-dependent part of the Hamiltonian as a rotating field in the $\hat{S}^{x}, \hat{S}^{y}$ plane:

$\hat{V}_{a}(t)=-\frac{V_{g}}{L} \int d^{3} r z \theta(L / 2-|z|)\left[e^{-i \Omega t} \hat{S}^{+}(\mathbf{r})+\right.$ H.c. $]$.

Here, $\hat{S}^{+}(\mathbf{r})=\hat{\Psi}_{\uparrow}^{\dagger}(r) \hat{\Psi}_{\downarrow}(r), \theta(z)$ is the Heaviside step function, and $L$ is the height of the box potential. $V_{g}=m g L\left(\mu_{\uparrow}-\mu_{\downarrow}\right) /\left(\mu_{\uparrow}+\mu_{\downarrow}\right)$, where we use Eq. (3) to tune a flat static potential.

\section{B. Heating rate}

The heating rate due to irreversible transitions induced by the residual time-dependent term (4) in the effective potential can be calculated from the linear response of the system to this perturbation. For this purpose, it is convenient to rewrite the time-dependent perturbation in momentum space:

$$
\hat{V}_{a}(t)=\sum_{q} v_{q} e^{-i \Omega t} \hat{S}_{q}^{+}+\text {H.c. }
$$

where $\hat{S}_{q}^{+}=\int d^{3} r \hat{S}^{+}(\mathbf{r}) e^{-i \mathbf{q} \cdot \mathbf{r}}$ and $v_{q}$ is the spatial Fourier transform of the time-dependent effective potential:

$v_{q}=-i V_{g} \delta_{q_{x}, 0} \delta_{q_{y}, 0} \frac{L q \cos (L q / 2)-2 \sin (L q / 2)}{(L q)^{2}} e^{-\left(\lambda_{0} q\right)^{2}}$.

The Gaussian falloff at large $q$ is due to convolution with the optical resolution that limits the sharpness of the potential features. In our experiment, the resolution $\lambda_{0} \approx 3 \mu \mathrm{m}$ happens to be close to the Fermi wavelength $\lambda_{F} \approx 2.6 \mu \mathrm{m}$, so for simplicity we identify the two scales and use $\lambda_{F}$ also as the resolution limit.

The transition rate $\Gamma_{q}$ induced by the perturbation at wave vector $q$ is directly related to the spectral function of the operator $\hat{S}_{q}^{+}$at the Rabi frequency through

$$
\Gamma_{q}=\left|v_{q}\right|^{2} \operatorname{Im}\left\langle\hat{S}_{q}^{+}(\Omega) \hat{S}_{-q}^{-}(-\Omega)\right\rangle_{\text {Ret }} .
$$

Without interactions, the transition rate is exponentially small in the high frequency $\Omega$, because the only way the perturbation can excite a resonant transition is to give the particle extremely high momentum $\hbar q \approx \sqrt{m \hbar \Omega}$, which is suppressed by the Gaussian resolution limit in the matrix element (6). In the presence of interactions, on the other 


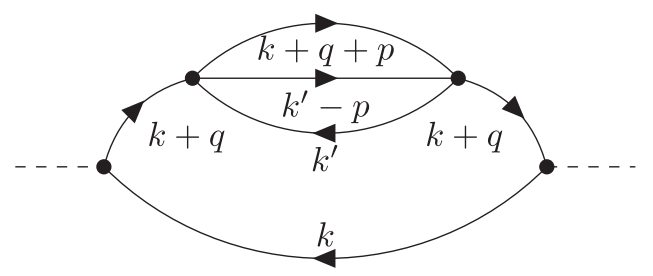

FIG. 2. Linear response to the residual time-dependent potential. In the diagram, the incoming and outgoing dashed lines represent the operators $\hat{S}_{q}^{+}$and $\hat{S}_{q}^{-}$attached at the vertices with the couplings $V_{q}$ and $V_{-q}$, respectively. The vertices connecting four solid lines represent the contact $s$-wave interactions.

hand, a transition can satisfy energy conservation even for small momentum transfer by utilizing the interaction term to create another particle-hole pair. The diagram of the lowest-order process that can lead to a transition is shown in Fig. 2. Pictorially, the perturbation creates a particle-hole pair with small momentum $q$ (and concomitantly low energy). The excited particle with momentum $k+q$ then scatters on another particle with momentum $k^{\prime}$ inside the Fermi sea in a collision with large momentum transfer $p \gg$ $k_{F}\left(k_{F}\right.$ is the Fermi wave vector) so that the pair of particles emerging from the collision are near the required final energy $\hbar \Omega$.

The transition rate in this process can be framed as a Fermi golden rule calculation, equivalent to the imaginary part of the diagram in Fig. 2:

$$
\Gamma_{q}=\frac{1}{\hbar} \sum_{f}|\langle f|\hat{T}| i\rangle|^{2} \delta\left(E_{f}-E_{i}-\hbar \Omega\right),
$$

where

$$
\hat{T}=\sum_{k} \frac{\hat{H}_{\text {int }}|k, q\rangle\langle k, q| v_{q} \hat{S}_{q}^{+}}{\hbar \Omega-E_{n}}
$$

Here, the intermediate states are the particle-hole states labeled by the hole momenta $k$ :

$$
|k, q\rangle=\hat{c}_{\uparrow, k+q}^{\dagger} \hat{c}_{\downarrow, k}\left|\psi_{\mathrm{FS}}\right\rangle,
$$

and the accessible final states consist of two particles and two holes with $S^{z}= \pm 1$, such as

$$
\left|k, k^{\prime}, p, q, s\right\rangle=\hat{c}_{s, k+q+p}^{\dagger} \hat{c}_{-s, k^{\prime}-p}^{\dagger} \hat{c}_{\downarrow, k^{\prime}} \hat{c}_{\downarrow, k}\left|\psi_{\mathrm{FS}}\right\rangle .
$$

The effective interaction vertex merits a brief discussion. By a slight abuse of notation, we write the contact interaction

$$
\hat{H}_{\mathrm{int}}=\frac{u}{\mathcal{V}} \sum_{k k^{\prime} p} \hat{c}_{\uparrow, k+p}^{\dagger} \hat{c}_{\downarrow, k^{\prime}-p}^{\dagger} \hat{c}_{\downarrow, k^{\prime}} \hat{c}_{\uparrow, k}
$$

using the same symbol used for the interaction Hamiltonian (1b), where $\mathcal{V}$ is the volume. However, it should be noted that the effective contact interaction is really the low-energy limit of the $T$ matrix. In most cases, it is given by the pseudopotential $u=4 \pi \hbar^{2} a / m$ with $a$ the $s$-wave scattering length. But at unitarity the dependence on the energy of the scattered states is important, as it cuts off the divergence at any nonvanishing energy. The $T$ matrix appropriate for the vertex in Fig. 2 from the Lippmann-Schwinger equation at unitarity is

$$
u=\frac{2}{\rho(\Omega / 2)}=\frac{8}{3 n} \frac{\epsilon_{F}^{3 / 2}}{\sqrt{\hbar \Omega / 2}},
$$

where $n$ is the particle density and $\rho(\epsilon)$ the single-particle density of states per unit volume (DOS). We also define an interaction energy scale $U=u n$.

Substituting into the Fermi golden rule expression, we obtain

$$
\Gamma_{q} \approx \frac{1}{\hbar} \sum_{k, k^{\prime}, p, s}\left(\frac{\left|v_{q}\right| U}{\hbar \omega+\xi_{k}-\xi_{k+q}}\right)^{2} n_{k} n_{k^{\prime}}\left(1-n_{k+q}\right)\left(1-n_{k+q+p}\right)\left(1-n_{k^{\prime}-p}\right) \delta\left(\xi_{k+q+p}+\xi_{k^{\prime}-p}-\xi_{k}-\xi_{k^{\prime}}-\hbar \omega\right) .
$$

The factors $n_{k}\left(1-n_{k+q}\right)$ constrain the sum over $k$ to a fraction of approximately $|q| / k_{F}$ of the Fermi sphere volume. Physically, this is the phase space available for creating the low-momentum particle-hole pair intermediate state. The other constraints are obeyed automatically due to the required large momentum transfer $p$ in the collision. In addition, because we assume $\hbar \Omega \gg \epsilon_{F}$, we neglect the small particlehole energy $\xi_{k}-\xi_{k+q}$ in the denominator and the hole energies $-\xi_{k}$ and $-\xi_{k^{\prime}}$ in the $\delta$ function. Thus, we obtain

$$
\Gamma_{q} \approx \frac{\left|v_{q}\right|^{2} U^{2}}{4 \hbar(\hbar \Omega)^{2}} \frac{|q|}{k_{F}} \mathcal{V} \rho(\hbar \Omega)=\frac{3 N}{16 \hbar} \frac{\left|v_{q}\right|^{2} U^{2}}{\left(\hbar \Omega \epsilon_{F}\right)^{3 / 2}} \frac{|q|}{k_{F}},
$$

where $N$ is the particle number. The total transition rate is the integral over $\mathbf{q}=q \hat{\mathbf{z}}$ up to the resolution cutoff $q_{0} \approx k_{F}$ :

$$
\Gamma \approx \frac{N}{\hbar} \frac{3}{64 \pi} \frac{V_{g}^{2} U^{2}}{\left(\hbar \Omega \epsilon_{F}\right)^{3 / 2}}\left(\frac{1}{k_{F} L}\right) \ln \left(\frac{k_{F} L}{\pi}\right) .
$$

The factor $\left(k_{F} L\right)^{-1}$ stems from the suppressed phase space for excitation of the Fermi sea with low-momentum transfer, while the logarithmic correction is due to the integration over the $1 / q$ behavior of the function $\left|v_{q}\right|^{2} q$ for $q \gg \pi / L$.

We remark that the scaling with $\Omega$ can be understood without a calculation by noting that the transition rate is a 
second-order process with the intermediate states constrained to low energy compared to $\Omega$ due to the momentum cutoff on the perturbation. Thus, the energy denominator due to the virtual transition is approximately $\Omega$, and the transition rate must scale as $\Gamma \sim\left[\left(V_{g} U\right) / \Omega\right]^{2} \rho(\Omega)$. Plugging in the DOS in $3 \mathrm{D}$, we get the correct scaling with $\Omega$. The small momentum transfer $q$ limits the phase space for intermediate states giving the $|q| / k_{F}$ factor in $\Gamma_{q}$.

Finally, we can convert the transition rate to a heating rate by multiplying it with the average energy increase per particle per unit time. In our experiment, the energy $\hbar \Omega / 2$ given to the excited particle pair is sufficient to escape the trap. Therefore, the heating is due to the holes left in the Fermi sea, leading to the heating rate $\dot{\epsilon}=\epsilon_{F} \Gamma / N$. To get a dimensionless measure of the heating rate, we define $\eta=\hbar \dot{\epsilon} / \epsilon_{F}^{2} . \eta$ gives the relative energy change per particle $\delta \epsilon / \epsilon_{F}$, which occurs in the characteristic timescale of the system $\tau_{F}=\hbar / \epsilon_{F}$. If $\eta \ll 1$, we can observe phenomena slow on the scale of $\epsilon_{F}$ without being affected by the heating. At unitarity, we get

$$
\eta=\frac{2}{3 \pi}\left(\frac{V_{g}}{\hbar \Omega}\right)^{2} \sqrt{\frac{\epsilon_{F}}{\hbar \Omega}} \frac{1}{k_{F} L} \ln \left(\frac{k_{F} L}{\pi}\right) .
$$

In our experiment, we can reach driving frequencies $\Omega / 2 \pi>10 \mathrm{kHz}$ where this parameter is $\eta<10^{-4}$, so heating is not expected to be a problem. Before proceeding, we note that a semiclassical calculation of the heating induced by periodic forces acting on an ultracold gas is reported in Ref. [46].

\section{EXPERIMENT}

Our experiments are performed with a quantum degenerate gas of ${ }^{40} \mathrm{~K}$ atoms, prepared in an incoherent, spinbalanced mixture of the two lowest-energy states, $|\downarrow\rangle=$ $|9 / 2,-9 / 2\rangle$ and $|\uparrow\rangle=|9 / 2,-7 / 2\rangle$, with the notation $\left|F, m_{F}\right\rangle$. The flat trap $V_{\text {trap }}$ is created by three laser beams with a wavelength of $532 \mathrm{~nm}[38,47]$ (see Fig. 1); a "tube" beam is created by a wide Gaussian beam $(125 \mu \mathrm{m}$ waist radius) that has a circular hole at its center, created by a digital mirror device [48]. The other two "end-cap" beams are created by two highly elliptical Gaussian beams with waist radii of 5.5 and $180 \mu \mathrm{m}$. Together, they generate a dark cylindrical volume with an approximate height of $39 \mu \mathrm{m}$ and a diameter of $55 \mu \mathrm{m}$, defined by the full width at half maximum of the atomic density. The cylinder symmetry axis is parallel to the gravitational force.

The experimental sequence starts by cooling the gas to quantum degeneracy in a crossed optical dipole trap [49]. To improve the loading efficiency into the flat trap, we add a second crossing beam to the optical trap described in Ref. [49]. This yields a harmonic trap with trapping frequencies of $\omega_{r}=2 \pi \times 236(1) \mathrm{Hz}$ and $\omega_{z}=2 \pi \times 27(2) \mathrm{Hz}$, in the radial and axial directions, respectively. After forced evaporation, there are $N \approx 5 \times 10^{5}$ atoms at $T / T_{F} \approx 0.24$ in this trap, where $N$ is the total atom number in both spin states and $T_{F}$ the Fermi temperature.

To load the flat trap, the tube beam is ramped to $30 \mathrm{~mW}$ already at the beginning of the evaporation in the harmonic trap. The magnetic-field gradient that counteracts gravity is ramped to its final value, as given in Eq. (3), in $0.5 \mathrm{~s}, 1 \mathrm{~s}$ before the harmonic trap is turned off. In our system, we have an additional undesirable small magnetic gradient of $\left[\left(d B_{z}\right) / d y\right] \approx 0.68 \mathrm{G} / \mathrm{cm}$ in the transverse direction, which we compensate with another pair of coils. The sequence continues with a ramp-up of the caps and tube beam power to 50 and $150 \mathrm{~mW}$, respectively. The two traps are held overlapping for $50 \mathrm{~ms}$, and then the harmonic trap is ramped down in $200 \mathrm{~ms}$. We typically load around $32 \%$ of the atoms into the flat trap. Finally, the atoms are cooled in the flat trap by evaporation, forced by ramping down the power of the caps and tube beams in $2 \mathrm{~s}$ to 20 and $50 \mathrm{~mW}$, respectively. To ensure the cloud has reached equilibrium, we wait for an additional $0.8 \mathrm{~s}$ before performing a measurement. The final typical conditions in the flat trap with the rf field are $N \approx 60 \times 10^{3}$ atoms with $T / T_{F} \approx 0.15$. The typical Fermi energy $\epsilon_{F} / h \approx 940 \mathrm{~Hz}$ is determined independently from an in situ density measurement of the gas (see the Appendix A). The magnetic field is tuned around the Feshbach resonance, at $202.14 \mathrm{G}$, determining the strength of interactions, $1 / k_{F} a$. It is ramped adiabatically to its final value in $10 \mathrm{~ms}$, where it is typically kept for $400 \mathrm{~ms}$. In the last $200 \mathrm{~ms}$ of the experimental sequence, the rf pulse is turned on with a typical Rabi frequency of around $\Omega / 2 \pi \approx 10.5 \mathrm{kHz}$.

\section{RESULTS}

\section{A. Relaxation dynamics}

Prior to turning the rf field on, the atomic densities of the two spins are not uniform [Fig. 3(a)], because the magnetic-field gradient given by Eq. (3) overcompensates (undercompensates) gravity for state $|\downarrow\rangle(|\uparrow\rangle)$ by $5.9 \%$. Once the resonant rf field is turned on, the densities start to equilibrate. We study this relaxation process by preparing the gas with only spin $|\downarrow\rangle$ atoms and imaging them from the side of the cylinder after different waiting times [Figs. 3(a)-3(c)]. To quantify the nonuniformity of the gas, we plot the slope of the optical depth (OD) at the center of the trap, normalized by its maximal value at $t=0$ (main part of Fig. 3). The gas relaxes to a uniform density with damped oscillations. The oscillation frequency is roughly given by the time it takes an atom with a Fermi velocity to traverse the trap height back and forth. The density reaches a steady state for rf pulse duration longer than $100 \mathrm{~ms}$. The residual density inhomogeneity due to the finite steepness and imperfections of the trapping potential is discussed in Appendix A. 


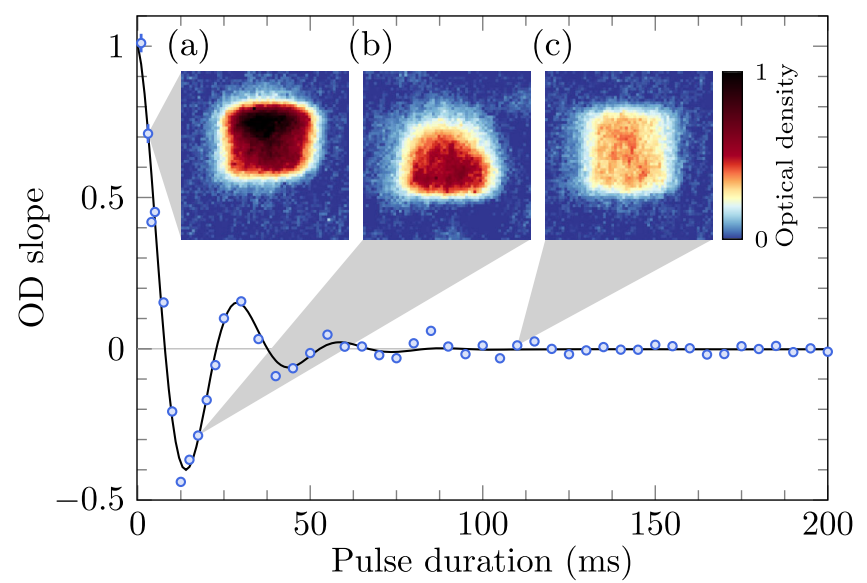

FIG. 3. Relaxation to a uniform density. The gas is prepared in a spin-polarized $|\downarrow\rangle$ state without the rf field. The magnetic field is set according to Eq. (3), overcompensating gravity for this state by $5.9 \%$. At $t=0$, the $\mathrm{rf}$ field with $\Omega / 2 \pi \approx 15.7 \mathrm{kHz}$ is switched on and flattens the time-average potential. The in situ density of gas is recorded from the side of the cylindrical trap after variable rf pulse duration (insets showing 3, 17.5, and $110 \mathrm{~ms}$ ). The color bar represents the optical depth (OD) in the absorption image. The main figure shows the density relaxation dynamics, quantified by the average change in the OD from side to side of the image. The error bars represent $1 \sigma$ deviation from linear fit to the OD slope. We fit the data with $g(t)=a e^{-t / \tau} \cos (2 \pi f t+\phi)+b$ (solid line) and obtain $\tau=16(1) \mathrm{ms}$ and $f=33.6(7) \mathrm{Hz}$. The reduction of the $\mathrm{OD}$ for longer pulse duration is due to decoherence to a spin-balanced mixture while measuring atoms only in the $|\downarrow\rangle$ state (see the main text). This measurement is done at a magnetic field of approximately $203 \mathrm{G}$, where the scattering length between states $|\downarrow\rangle$ and $|\uparrow\rangle$ is $a \approx-1152 a_{0}\left(a_{0}\right.$ is the Bohr radius).

Since spin-polarized fermions do not interact via $s$-wave scattering, a question that may arise is how the relaxation process actually occurs. While the rf rotation creates a $|\uparrow\rangle$ component on a short timescale of the inverse Rabi frequency, the gas remains spin polarized, albeit with the spin oriented in a different direction. However, small spatial inhomogeneities in the rf and magnetic fields lead to spin dephasing and eventually, through atomic diffusion, also to decoherence. Therefore, the spin-polarized gas becomes a balanced spin mixture on a relatively short timescale of $10 \mathrm{~ms}$ [50]. Thanks to the invariance of the interaction Hamiltonian, the rf resonance frequency does not change as the gas transforms from being noninteracting to strongly interacting [45].

\section{B. Momentum distribution of the uniform gas}

An important issue to consider is heating, which may occur during the initial relaxation phase and during the continuous operation of the rf pulse. We obtain the temperature of the gas by measuring its momentum distribution. Ordinarily, this is done by letting the gas expand ballistically either in free space or in a harmonic trap [51]. Because of the relatively large initial size of the cloud, the free expansion requires particularly long expansion times, which are not always feasible. Expansion in a harmonic trap, on the other hand, is done for a quarter of the trap period but is sensitive to anharmonicity of the trap $[40,41,51,52]$.

Here, we take a different approach and use Raman spectroscopy, which has the advantage that it can be applied to a trapped gas [53,54]. The technique relies on a linear relation between the two-photon Raman detuning and the velocity of the atoms which are transferred from state $|\uparrow\rangle$ to the initially unoccupied state $|9 / 2,-5 / 2\rangle$. In the experiment, the two Raman beams are pulsed after the application of a 200-ms-long rf pulse. By scanning the relative frequency between the beams, we obtain a spectrum that is directly proportional to the one-dimensional momentum distribution [53]. A typical result with dynamically driven uniform Fermi gas is shown in Fig. 4. The number of atoms in state $|9 / 2,-5 / 2\rangle$ is measured by selectively capturing them in a magneto-optical trap (MOT) and recording their fluorescence $[49,53]$. To improve the detection, we separate the wavelength of the MOT, which is close to the $D_{2}$ transition, from that of the collected scattered photons [55]. To this end, we add a dedicated probe beam, tuned to the $D_{1}$ transition, and filter the recorded image with an ultranarrow, 1-nm, optical bandpass filter [56]. The intensities of the two Raman beams are actively stabilized and programmed to follow a 1-ms-long Blackman pulse [57]. The one-photon Raman detuning is around $46.1 \mathrm{GHz}$ below the $D_{1}$ transition. To reduce unwanted single-photon scattering, which constitutes most of the background signal, we incorporate a temperature-stabilized etalon after the Raman laser to filter the broadband-amplified spontaneous emission.

We analyze the momentum distribution by fitting it with three different models (see Fig. 4). The first one is a numerical local-density approximation model of a gas in a realistic flat trap that is used in our experiment (solid blue line). In this model, we account for the finite steepness of the trap walls, which is calibrated using in situ density images (see Appendix A). The free parameters are the reduced temperature $\left(T / T_{F}\right)$ and the background offset. For comparison, we fit the data with two ideal models of harmonically trapped gas (dashed red line) [53] and ideal uniform gas (dotted black line). The doubly integrated momentum distribution of the latter is given by

$$
n\left(k_{z}\right)=\pi \frac{T}{T_{F}} \ln \left(1+\zeta e^{-\left(k_{z}^{2} / k_{F}^{2}\right) /\left(T / T_{F}\right)}\right),
$$

where $k_{z}$ is in the direction of the two-photon momentum transfer [53] and $\zeta$ is the fugacity with the implicit form $\mathrm{Li}_{3 / 2}(-\zeta)=-[4 /(3 \sqrt{\pi})]\left(T / T_{F}\right)^{-3 / 2}$, with $\operatorname{Li}_{n}(z)$ being the polylogarithm function. Notice that, due to the double 


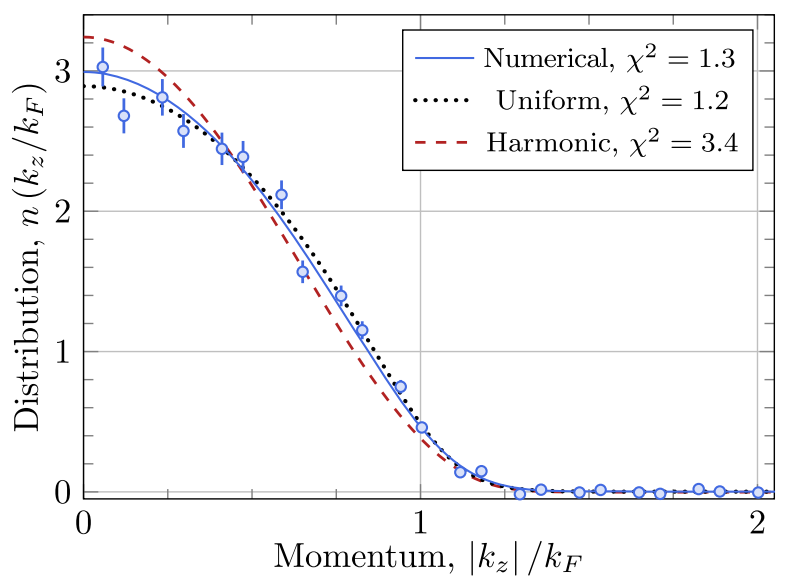

FIG. 4. The one-dimensional momentum distribution of a uniform periodically driven Fermi gas. The distribution, measured by Raman spectroscopy [53], is fitted with three models: a numerical model that provides a realistic description of the flat trap in the experiment (solid blue line; see Appendix A), an ideal homogeneous Fermi-Dirac distribution given by Eq. (18) (dotted black line), and a harmonically trapped gas (dashed red line). The numerical and homogeneous models yield a better fit to the data than the harmonic one, based on the $\chi^{2}$ values. The differences between the uniform and numerical models are not significant. The temperature extracted from the numerical model is $T / T_{F}=$ 0.15 (1) with $T=7(2) \mathrm{nK}$. The results of the other fits are discussed in the main text. This measurement is performed at a magnetic field of $B=209.18 \mathrm{G}$, where the atoms are very weakly interacting. The rf pulse duration is $200 \mathrm{~ms}$ with a Rabi frequency of $\Omega / 2 \pi \approx 10.5 \mathrm{kHz}$. Error bars represent one standard deviation of the measured values.

integration, there is no sharp Fermi surface in this functional even at $T=0$.

The numerical and uniform models fit the data markedly better than the harmonic one, as evident by comparing their $\chi^{2}$ fit values (see the legend in Fig. 4). The temperature extracted from the numerical model is $T / T_{F}=0.15(1)$ with $T=7(2) \mathrm{nK}$. The uniform model yields a very similar result of $T / T_{F}=0.16(2)$. In contrast, the harmonic model gives a much lower temperature of $T / T_{F}=0.08(2)$. In the numerical model, $k_{F}$ is calculated directly from in situ density images (see Appendix A), while in the uniform and harmonic models, it is left as a free fitting parameter. We find that the $k_{F}$ extracted from the uniform model is only $6(2) \%$ higher than the one calculated directly, while that of the harmonically trapped model is higher by $30(2) \%$.

To test whether the rf-induced spin rotation causes heating, we repeat the Raman measurement at different rf pulse durations, shown in Fig. 5. Within the experimental accuracy, we do not observe an increase of the temperature. This result is corroborated by the measurements of the condensate fraction versus time, described below. We therefore conclude that with our experimental parameters, satisfying $\Omega \gg \epsilon_{F} / \hbar$, the heating rate is too small to be detected.

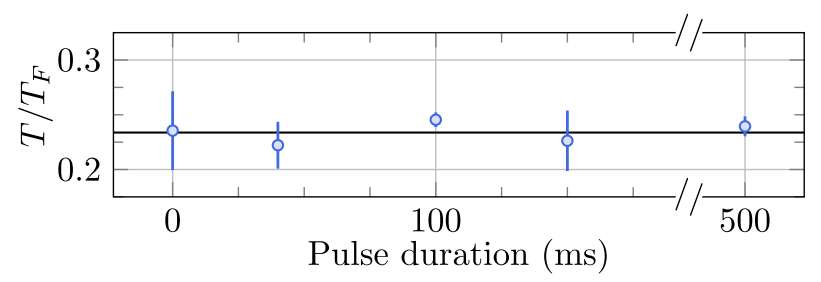

FIG. 5. Reduced temperature as a function of the rf pulse duration. The temperature is extracted from one-dimensional momentum distribution measurements fitted by a homogeneous Fermi-Dirac distribution. We observe no increase in $T / T_{F}$ up to $500 \mathrm{~ms}$, and this holds true also for $T$ alone. Note that the data in this figure are taken at slightly different conditions than the one in Fig. 4, with $N \approx 131 \times 10^{3}$ atoms at $T / T_{F} \approx 0.23$. The measurement is performed at a magnetic field of $B=209.18 \mathrm{G}$, where the atoms are very weakly interacting. The Rabi frequency is $\Omega / 2 \pi \approx 10.5 \mathrm{kHz}$. Error bars represent one standard error of the fit.

\section{Pair condensation}

We now turn to probe the many-body properties of a dynamically driven Fermi gas. When a spin-balanced Fermi gas is cooled below the critical temperature $T_{c}$, atoms with opposite spins pair and condense, forming a fermionic superfluid [26,58-60]. The value of $T_{c}$ depends on the interaction strength. The survival of superfluidity is a stringent test of our Floquet engineering scheme, since this phase is extremely sensitive to heating and differential forces acting on the spins.

In these experiments, we cool the gas below the superfluid transition at unitarity $\left(1 / k_{F} a \approx 0\right)$. At the end of the cooling stage, the magnetic field is ramped in $10 \mathrm{~ms}$ from $203.5 \mathrm{G}$ (weak interactions) to unitarity. There, it is held for $5 \mathrm{~ms}$, during which the atoms pair up and condense. The magnetic-field gradient and rf pulse are present during the last $200 \mathrm{~ms}$, long enough to ensure equilibrium. Since during this time the magnetic field is changing, we program the $\mathrm{rf}$ frequency to track the resonance transition.

We characterize the survival of the superfluid phase by measuring the condensate fraction, using the pairprojection technique $[59,61]$. To this end, the trap is abruptly turned off and at the same time the magnetic field is ramped rapidly ( $40 \mu \mathrm{s}$ ) to the BEC side of the resonance (199.8 G) [59]. This procedure projects the loosely bound pairs onto tightly bound molecules. We then let the gas expand for $24 \mathrm{~ms}$ and measure the distribution using absorption imaging. For the imaging to work, we dissociate the molecules by ramping back the magnetic field to unitarity just before taking the image. When the gas is superfluid, the recorded density distribution is bimodal, with condensed pairs appearing as a pronounced central peak (see Appendix B).

In Fig. 6, we plot the condensate fraction (lower) and total number of atoms (upper) at unitarity, extracted from the images of the expanded gas, as a function of the driving 


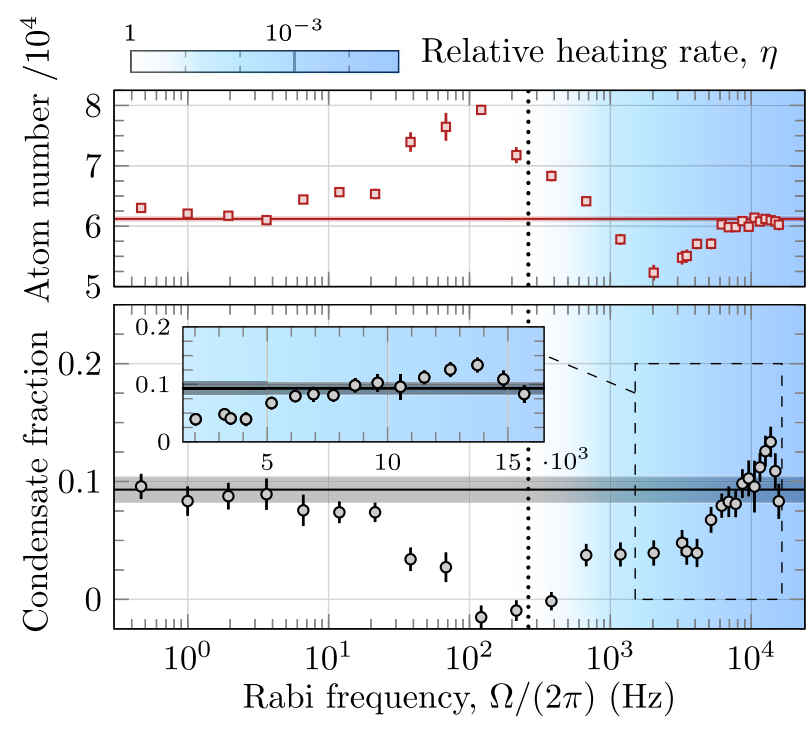

FIG. 6. Condensate fraction and total atom number at unitarity as a function of the driving frequency. At very low frequencies, the spin oscillation is slow enough such that both observables are the same as without the driving, which are marked by horizontal lines with shading representing the uncertainty. At higher frequencies, the driving has an adverse effect on the superfluid. The vertical dashed line marks the frequency at which $\eta=1$ [see Eq. (17)]. Above it, heating starts to be suppressed (the color represents the value of $\eta$ ). At even higher frequencies, the atom number returns to its initial value, and the condensate fraction reaches an even higher value than for a stationary gas (inset). The conditions are measured after $5 \mathrm{~ms}$ at the Feshbach resonance magnetic field, and the Rabi frequency is varied by changing the rf pulse power. The observables extraction procedure is discussed in Appendix B. The error bars combine the $1 \sigma$ mean confidence interval of the fit with statistical errors over ten repetitions.

frequency. The overlap between the two spin distributions is large enough even without the rf field to yield a condensate fraction of around 0.11 . We distinguish between three frequency regimes with qualitatively different behavior. At very low frequencies, we observe a quasistatic behavior with local equilibrium and no apparent change in the conditions of the gas. As the frequency is increased, we cross to the second regime, where the driving generates spin currents and micromotion that clearly harms the superfluid. Initially, at around $\Omega \sim 100 \mathrm{~Hz}$, the heating does not lead to a loss of atoms, because the energy of the excitation is smaller than the trap depth. In fact, as the condensate fraction decreases, the number of detected atoms increases. This surprising behavior is a result of a partial correlation between the number of detected atoms following the pair-projection technique and the number of pairs. It exists since during the magnetic field ramping a small fraction of the pairs are lost, most likely to deeply bound molecular states. At higher frequencies, atoms have enough energy to leave the trap, but heating is gradually suppressed due to the scaling with $\Omega$ of the second-order process (see Sec. II). In the third regime, defined by $\eta<1$,

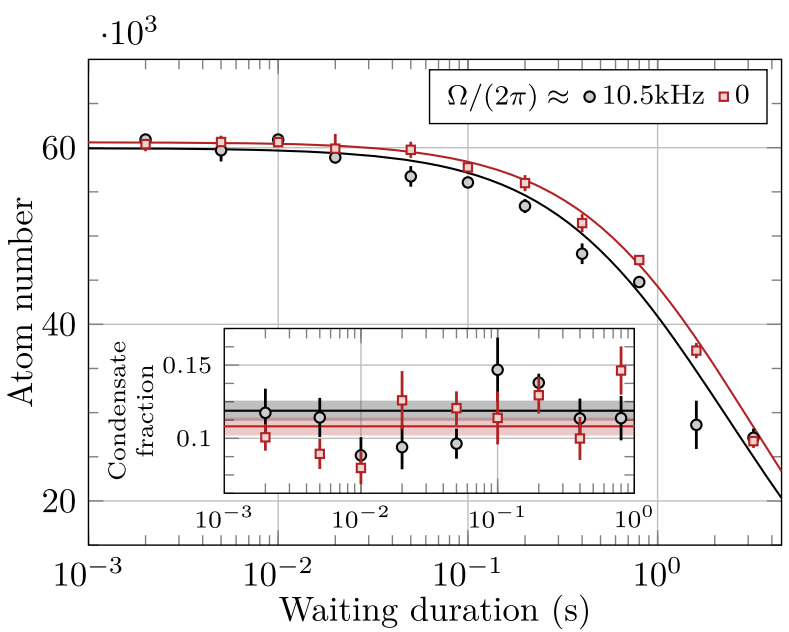

FIG. 7. Time dependence of the atom number and condensate fraction at unitarity. Data are taken after different waiting durations both with (black circles) and without (red squares) the rf driving. In both cases, the loss has a similar trend, which is well fitted by the model in Eq. (19) (solid lines). Inset: The condensate fraction (same marks) is plotted together with the weighted average (solid lines), and its standard deviation (shades) shows no decrease. Error bars are determined as in Fig. 6.

the loss decreases and the condensate revives. In the highfrequency limit, where $\eta \ll 1$, the atom number returns to its initial value while the condensate fraction reaches an even higher value, an effect we attribute to a better spatial overlap between the spins in a uniform gas.

We now return to the question of heating at high driving frequency. As shown in Fig. 5, we do not observe a rise of the temperature, as extracted from the momentum distribution. However, in the superfluid phase, the condensate fraction is a much more sensitive thermometer. In Fig. 7, we plot the total number of atoms and condensate fraction versus the waiting time at unitarity (black circles). We employ an rf field with a relatively high Rabi frequency $(\Omega / 2 \pi \approx 10.5 \mathrm{kHz})$, where the density is already uniform and the condensate fraction reaches its high value (see Fig. 6). To distinguish between loss and heating due to the rf driving and other sources, we repeat this measurement without the rf field (red squares). The data, taken with a waiting time of up to more than $1 \mathrm{~s}$, do not point to any heating, as there is no reduction of the condensate fraction, even when the total number of atoms has decreased by approximately a factor of 2 .

The decay in the number of atoms is almost identical with or without the rf pulse. We analyze the data using the following loss model [62-64]:

$$
\frac{d n}{d t}=-K_{1} n-K_{2} n^{2}-K_{3} n^{3}
$$

where $n$ is the total atomic density. $K_{1}=1 / 13.5 \mathrm{~s}^{-1}$ is the single-body loss rate, determined by the rate of collisions 
with the residual gas in the vacuum chamber, and measured independently. $K_{2}$ and $K_{3}$ are the two- and three-body loss rate coefficients, respectively. Previously, these parameters were measured only with harmonically trapped gases, which complicates the analysis due to the nonlinear density dependence in this model. Here, we benefit directly from the uniformity of the gas and from the fact that its shape and volume are almost unchanged as the atom number diminishes. Fitting the data taken with the rf pulse with both coefficients as free parameters (black solid line) yields $K_{3}=9(1) \times 10^{-25} \mathrm{~cm}^{6} \mathrm{~s}^{-1}$ and $K_{2}=0$. This shows that the loss is mainly due to three-body recombination. Since in the data without the rf pulse the density is not homogeneous and, in fact, differs between the two spin components, we do not use it to extract loss coefficients. Qualitatively, however, it is still fitted well by the model of Eq. (19) (red solid line). Our value for $K_{3}$ is 10 times higher than the one measured in a harmonic trap and at a significantly higher temperature in Ref. [63]. We note, however, that their maximal value of $K_{3}$, which is measured on the BEC side of the resonance, agrees with our measurement at unitarity.

\section{The contact parameter of a uniform gas}

We now turn to a measurement of the contact parameter in the BEC-BCS crossover regime. This parameter is central to a set of universal thermodynamic and energetic relations [26,65-69], many of which have been tested experimentally [70-73]. Previous works determine the value of the contact with harmonically trapped gas at different temperatures and interaction strengths [70,74,75]. Local measurements resolve the contact of a quasihomogeneous sample [33,35,36,76-79]. Until now, the contact of a truly uniform gas was measured only at unitarity [80].

We determine the contact from the power-law tail of $\mathrm{rf}$ line shapes taken with the uniform gas $[33,49,70,80]$. In contrast to the condensate fraction experiments, where the condensate is formed after approximately $2 \mathrm{~ms}$, here we observe that it takes at least $100 \mathrm{~ms}$ for the tail to fully develop. For this reason, we wait for $400 \mathrm{~ms}$ in the final magnetic field before measuring the rf line shape. The number of atoms and temperature are similar to the condensate fraction experiments. The spin-rotation rf field, with $\Omega / 2 \pi \approx 10.5 \mathrm{kHz}$, is turned on for the last $200 \mathrm{~ms}$. It is turned off $0.5 \mathrm{~ms}$ before we probe with a $1-\mathrm{ms}$ square pulse of a second rf field, whose frequency we scan near the $|\uparrow\rangle \rightarrow|9 / 2,-5 / 2\rangle$ transition. The atom number in state $|9 / 2,-5 / 2\rangle$ is again detected with fluorescence imaging [49]. A typical rf line shape is shown on a logarithmic scale in the inset in Fig. 8. A universal power-law tail over two decades is clearly visible. To extract the contact, we work in natural Fermi units and normalize the spectrum to $1 / 2$. The tail is then fitted with $C /\left(2^{2 / 3} \pi^{2} \nu^{3 / 2}\right)$ (black line in the inset), where $C$ is the contact parameter in units of $N k_{F}$. Owing to the high sensitivity of our fluorescence detection

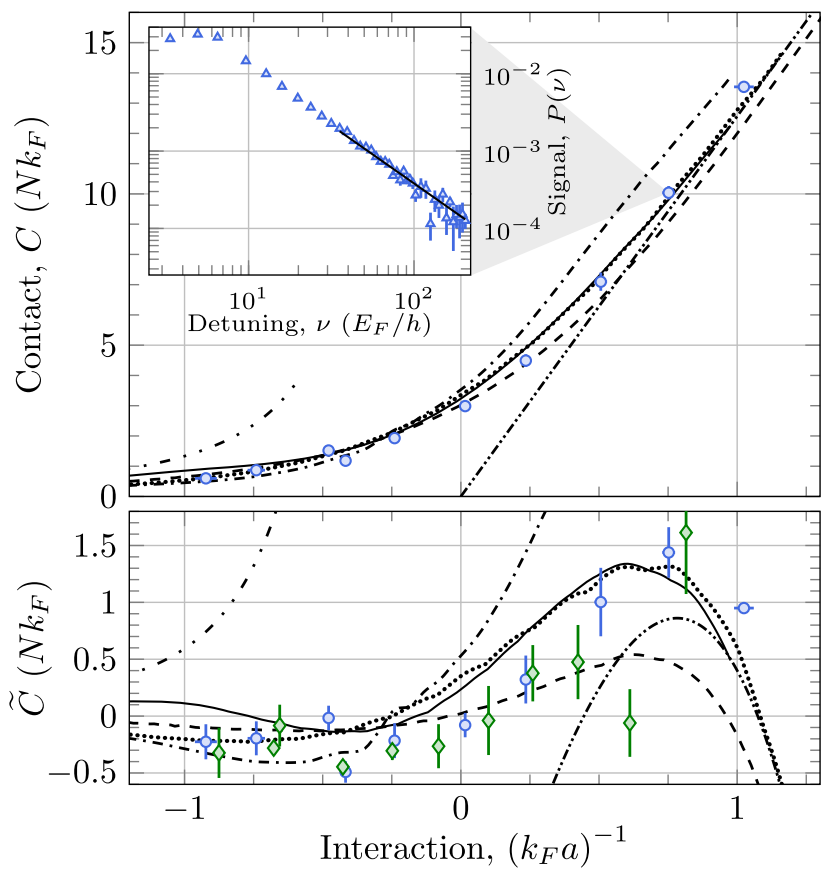

FIG. 8. The contact of a uniform Fermi gas in the BEC-BCS crossover. The contact is extracted from the tail of rf line shapes taken at different interaction parameters. As an example, the inset shows the line shape at $\left(k_{F} a\right)^{-1}=0.75(2)$ together with its fit (black solid line). Upper: The theoretical prediction for the contact in the BCS (BEC) limit is shown as a loosely (densely) dash-double-dotted line. We compare our data (blue circles) with a non-self-consistent $T$-matrix model at $T=T_{c}$ improved by Popov (dash-dotted line) and at $T=0$ (solid line) [81], a Luttinger-Ward calculation (dashed line) [82], and a GPF calculation (dotted line) [83,84]. The FNDMC line [78,85] is indistinguishable from the GPF line on this scale. Lower: For a better quantitative comparison, we define a shifted contact as $\tilde{C}=C-3 \exp \left(1.4 / k_{F} a\right)$. In this plot, we also add previous measurements done with a quasihomogeneous gas (green diamonds) [35]. Error bars represent $1 \sigma$ confidence interval of the fit.

scheme, we keep the rf power constant for all detunings, while the maximal transferred fraction is no more than $8 \%$. The systematic error in the determination of the contact due to the remaining density inhomogeneity in our realization of the flat trap is estimated by calculating the densityweighted average of a theoretical contact [81] using our calibrated model of the trap (see Appendix A). We find that the density-averaged contact, representing our measured contact, is lower than the homogeneous contact by $5 \%$ in the BCS side $\left(1 / k_{F} a=-1\right)$ and higher by $7 \%$ in the BEC side $\left(1 / k_{F} a=1\right)$. Near unitarity, the large scattering length reduces the deviation to less than $1 \%$. As a comparison, for harmonically trapped gas at the same average density, the systematic errors are $19 \%$ and $25 \%$ in the BCS and BEC sides, respectively.

In Fig. 8, we plot the contact of a uniform Fermi gas at various interaction strengths in the BEC-BCS crossover. Starting from the BCS side $(a<0)$, the contact increases 
monotonically toward the BEC side of the crossover, where it converges to the asymptotic behavior of a molecule: $C_{\mathrm{BEC}}=4 \pi / k_{F} a$ [82]. We find that, already above $1 / k_{F} a \approx 0.8$, our data are very close to $C_{\mathrm{BEC}}$. In contrast, the weak-coupling BCS limit of the contact, $C_{\mathrm{BCS}}=4\left(k_{F} a\right)^{2} / 3$ [82], is not attained even at $1 / k_{F} a=-1$. We compare our data to several theories and previous measurements. On the BEC side, there is a pronounced difference between the $T=0$ and $T=T_{c}$ predictions [81]. Our data, which are taken slightly below $T_{c}$, agree with the $T=0 T$-matrix calculation. We also find a good agreement with the Gaussian pair fluctuation (GPF) calculation $[83,84]$ and fixed-node diffusion Monte Carlo simulation (FNDMC) $[78,85]$, especially in the $\mathrm{BEC}$ region. A Luttinger-Ward calculation [82] is slightly below our data on the BEC side. Close to unitarity $\left[1 / k_{F} a=-0.02(1)\right]$, our measured $C=2.99(11)$ is in good agreement with values measured using $\mathrm{rf}$ spectroscopy $[C=3.07(6)]$ [80], Bragg spectroscopy $[C=2.95(14)]$ [36], and impurity loss $[C=3.09(34)]$ [79]. It is slightly lower than $C=3.51(18)$ [76] and $C=3.37(4)$ [78] obtained from in situ thermodynamic measurements. Similar data taken in the BEC-BCS crossover, albeit with a quasihomogeneous gas and above $T_{c}$, are in agreement with ours, to within the experimental accuracy [35].

\section{DISCUSSION}

In this work, we have demonstrated that Floquet engineering can be used with a continuous interacting Fermi gas without affecting its intrinsic many-body behavior. Specifically, we have employed the technique to eliminate the effect of a spin-dependent potential and achieve a flat trap. Our experiments are done with a driving frequency that is much higher than all other relevant experimental scales. In this regime, we have found no detectable heating during the experiment due to periodic driving. Measurements of the condensate fraction and contact parameter show the same behavior as expected in a stationary uniform Fermi gas. Furthermore, our dynamical levitation scheme can be used to generate a uniform density of other spin mixtures.

The full Hamiltonian of Eq. (1) depends explicitly on time and, therefore, does not conserve energy. In contrast to many-body localized systems [86-89], our gas is ergodic, and, thus, it is not protected from heating [16-18]. Nevertheless, interacting many-body systems can attain long-lived prethermal states, following a quench of the Hamiltonian parameters [90-94] or upon initiation of periodic driving $[20,21,95,96]$. In particular, discrete lattice systems show an exponentially slow energy absorption rate at high frequencies leading to Floquet prethermal states that persist for a time exponentially long with the drive frequency $\Omega[19-23,25]$. The Fermi gas in the continuum does not benefit from such exponential suppression of the heating rate. Nonetheless, we have shown in Sec. II that the smooth spatial structure of the periodic perturbation leads to parametric suppression of the heating rate in a unitary Fermi gas. The suppression is controlled by the small values of $V_{g} /(\hbar \Omega)$, with $V_{g}$ being the strength of the timedependent perturbation, $\epsilon_{F} /(\hbar \Omega)$, and $1 / k_{F} L$. This allows us to obtain prethermal states with lifetimes much longer than the characteristic many-body timescale. In this work, we have focused on demonstrating this fact with measurements of the momentum distribution, the contact parameter, and the condensate fraction. It will be interesting to investigate the heating process and thermodynamic properties of the driven gas in the future.

Our work should also be placed in the framework of quantum information processing, where ultracold atoms are proposed as a resource for quantum memory [97-99]. A common cause of decoherence is spatially inhomogeneous spin-dependent potentials. As an example, the energy difference between two internal states of optically trapped atoms usually varies in position due to differential light shifts. In a classical ensemble, each atom can be treated independently with the rest of the ensemble acting as a fluctuating bath $[100,101]$. These fluctuations lead to decoherence of the qubit stored in this atom. Dynamical decoupling [102,103], a generalization of the celebrated Hahn echo technique [104], can substantially slow this relaxation process by applying multiple spin rotations [105].

Dynamical decoupling has been applied successfully in NMR [106-108], photonic systems [109], trapped ions [110,111], electron spin in solids [112-115], ultracold atoms [105,116], and Bose-Einstein condensates (BECs) $[117,118]$. In all cases, the decoupled system was weakly interacting and could be treated in a mean-field approach. Dynamical decoupling was not applied before to a strongly interacting ensemble with the aim of preserving its manybody behavior. In our work, the spin-dependent potential originates from the magnetic cancellation of gravity. The spin-rotation rf pulse we apply is a continuous version of a simple dynamical decoupling scheme. The absence of heating we observe is promising for future explorations of more sophisticated sequences tailored to generate specific local and global symmetries [24], for example, the realization of tilted Fermi-Hubbard chains where the rf dressing was used to tune a relative tilt difference of two different spin states [119].

\section{ACKNOWLEDGMENTS}

We thank Amir Stern, Ari Turner, Netanel Lindner, Keiji Saito, and David Huse for helpful comments. This research was supported by the Israel Science Foundation (ISF), Grants No. 1779/19 and No. 218/19, and by the U.S.-Israel Binational Science Foundation (BSF), Grant No. 2018264.

\section{APPENDIX A: NUMERICAL MODEL OF THE FLAT TRAP}

We develop a systematic approach to calibrate a numerical model for the flat trap potential. Using the numerical 


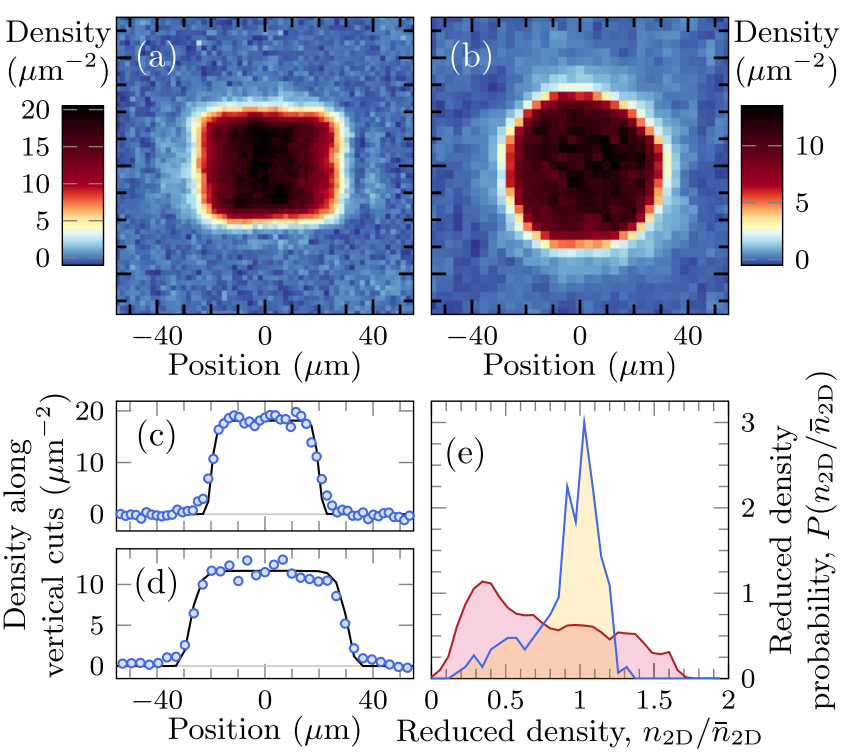

FIG. 9. In situ density analysis. An example of an in situ atomic density measured by absorption imaging from the side (a) and from the top (b) (the cylinder symmetry axis is vertical). Vertical cuts along the side and top images (circles) together with a fit to the numerical model (solid lines) are shown in insets (c) and (d), respectively. The flat part of the cut is where the density is uniform. Inset (e) shows a 2D density probability distribution of the flat trap (blue line) and a harmonically trapped gas (red line). The density probability function of the flat trap is peaked around the average density value, indicating that most of the density is close to the average. (a) and (b) are an average of five and ten experimental repetitions, respectively.

model, we calculate the Fermi energy and generate a momentum distribution functional used to fit the Raman spectrum (see Fig. 4). To this end, we simulate the density in the box trap using a model potential and fit it to the in situ integrated density, measured by absorption imaging. For this calibration, we create a spin-polarized gas at the same conditions as in the experiments presented in this paper. This is done by first preparing the gas in the flat trap in a spin-balanced configuration as described in the main text. Then, we apply an adiabatic rapid passage selectively from state $|\uparrow\rangle$ that drives the atoms from this state to a final $|9 / 2,+9 / 2\rangle$ state, leaving state $|\downarrow\rangle$ untouched. The force created by the magnetic gradient, initially working opposite to the gravity for the $|\uparrow\rangle$ state, flips its sign due to the change in the magnetic number $m_{F}$ and starts working in the direction of gravity, ripping the atoms from the flat trap through the lower cap wall. This procedure removes all of the atoms that are initially in state $|\uparrow\rangle$ while losing less than $10 \%$ from state $|\downarrow\rangle$. The magnetic-field gradient is set to perfectly cancel gravity for state $|\downarrow\rangle$, making the density homogeneous.

The next step is taking in situ absorption images of the spin-polarized gas (see Fig. 9). The OD of the gas is too high to image directly. To reduce the OD for imaging, we apply a sequence of two rf pulses. The first pulse transfers approximately $90 \%$ of the atoms from state $|\downarrow\rangle$ to state $|\uparrow\rangle$. The second pulse transfers all of the atoms from state $|\uparrow\rangle$ to state $|9 / 2,-5 / 2\rangle$, which is detuned by $92 \mathrm{MHz}$ from the optical transition. The last pulse ensures that no artifacts are introduced to the imaging due to a large atom number in off-resonant states. The two pulses are completed within less than $70 \mu \mathrm{s}$, ensuring the density is unchanged during this procedure.

The spin-polarized gas is essentially noninteracting and can be described by a Fermi-Dirac distribution. To fit the two-dimensional integrated density image, we calculate the density in a local density approximation [120]:

$$
n(r, z)=-\lambda_{\mathrm{dB}}^{-3} \mathrm{Li}_{3 / 2}(-\exp \{\beta[\mu-U(r, z)]\}),
$$

where $\lambda_{\mathrm{dB}}$ is the de Broglie wavelength, $\beta=1 /\left(k_{B} T\right)$ with $k_{B}$ being the Boltzmann constant, $U(r, z)$ is the trapping potential, and $\mu$ is the chemical potential, which is set by the total number of atoms. The model potential of the tube beam is parametrized by a power-law function, while the potential of the cap beams is taken as a Gaussian function:

$$
\begin{aligned}
U(r, z)= & U_{r}\left(r / \sigma_{r}\right)^{p} \\
& +U_{z}\left(\exp \left[-\frac{2\left(z-z_{0}\right)^{2}}{\sigma_{z}^{2}}\right]+\exp \left[-\frac{2\left(z+z_{0}\right)^{2}}{\sigma_{z}^{2}}\right]\right) .
\end{aligned}
$$

Here, $r$ is the radial coordinate relative to the symmetry axis of the tube (denoted by $z$ ), and $U_{r}$ is the potential barrier of the cylindrical wall. The tube radius $\sigma_{r}=32 \mu \mathrm{m}$ and the power-law exponent $p=13.6$ are extracted from a direct measurement of the laser beam that generates the potential [55]. $U_{z}$ is the potential barrier of the cap beams, and $\sigma_{z}=$ $7.5 \mu \mathrm{m}$ is their waist radii in the $z$ direction, also measured directly. $z_{0}=24 \mu \mathrm{m}$ is half the separation between the two cap beams, measured by imprinting the cap profile on a dilute expanded cloud of atoms [55]. The temperature $T$ is found self-consistently together with momentum distribution measurements. The only free parameters in the fit are $U_{r}, U_{z}$, and the center position of the fit. A twodimensional density function for fitting the in situ image is generated by integrating the three-dimensional density $n(r, z)$ along one of the Cartesian axes. An example of a typical calibration is shown in Fig. 9. Once the model potential is calibrated, we calculate $\epsilon_{F}$ from the peak density: $E_{F}=\left[6 \pi^{2} n_{F}(0,0)\right]^{2 / 3} \hbar^{2} / 2 m$. The density-averaged $\epsilon_{F}$ is smaller by $12 \%$. This difference is considerably smaller than in the harmonically trapped gas before loading the flat trap, in which the density-averaged $\epsilon_{F}$ is smaller by $44 \%$ than the peak $\epsilon_{F}$.

We quantify the density homogeneity in the form of a probability distribution function $P(n)$. We calculate the axially integrated probability distribution $P\left(n_{2 \mathrm{D}} / \bar{n}_{2 \mathrm{D}}\right)$ as an 
histogram of absorption images taken along the symmetry axis [Fig. 9(b), inset], where $n_{2 \mathrm{D}}$ is the two-dimensional atomic density and $\bar{n}_{2 \mathrm{D}}$ is its weighted average. The probability distribution is plotted in the inset in Fig. 9(e), together with the probability distribution of a harmonically trapped gas. A direct measure of uniformity is the standard deviation of $P\left(n_{2 \mathrm{D}} / \bar{n}_{2 \mathrm{D}}\right)$, which is 0.23 and 0.42 in units of $\bar{n}_{2 \mathrm{D}}$ for the flat and harmonic traps, respectively.

The knowledge of the trapping potential enables us to calculate the one-dimensional momentum distribution $n\left(k_{z}\right)$ and use it as a fitting function for the data acquired in the Raman spectroscopy experiment (Fig. 4). $n\left(k_{z}\right)$ is obtained by integrating the semiclassical distribution [120]

$$
f(\boldsymbol{r}, \boldsymbol{k})=\left\{\exp \left[\left(\frac{\boldsymbol{k}^{2}}{k_{F}^{2}}+\frac{U(r, z)}{E_{F}}-\frac{\mu}{E_{F}}\right) / \frac{T}{T_{F}}\right]+1\right\}^{-1}
$$

both spatially and along two momenta axes

$$
n\left(k_{z}\right)=\frac{4 \pi}{3 N} \frac{1}{(2 \pi)^{3}} \int f(\boldsymbol{r}, \boldsymbol{k}) d^{3} \boldsymbol{r} d k_{x} d k_{y} .
$$

\section{APPENDIX B: EXTRACTING THE CONDENSATE FRACTION}

To separate the thermal wings and the central peak, we image the cloud after a relatively long expansion. As a result, the absorption signal is weak. To improve the signalto-noise ratio, we employ a deep-learning approach to filter out the background noise in the images [121]. We verify that this noise removal procedure does not change significantly the reported CF values and only reduces the uncertainty. The recorded density can be roughly considered as dissociated pairs that have either nonzero or zero center-of-mass momentum. The latter are the condensed pairs which constitute the central peak of the image (see Fig. 10). Each of the two populations expands differently, according to their respective momentum distribution. The total atom number is extracted by a direct integration of the OD image, and the error bars indicate statistical standard error only.

Following the time of flight, the noncondensed part of the gas is characterized by a wider expansion with respect to the trap dimensions, while the condensed part spreads only slightly beyond its original size, set by the tube beam diameter [40]. To separate the condensed and noncondensed parts, we mask out the central region of the image (dashed line in Fig. 10) and fit only the tail of the azimuthally averaged signal with the momentum distribution of a thermal gas of noninteracting bosons [120] (pink shading in Fig. 10):

$$
n_{\mathrm{th}}(k)=\frac{V}{4 \pi^{2} \lambda_{T}} \mathrm{Li}_{1 / 2}\left(z e^{-\lambda_{T}^{2} k^{2} / 4 \pi}\right)+N_{\mathrm{bg}},
$$

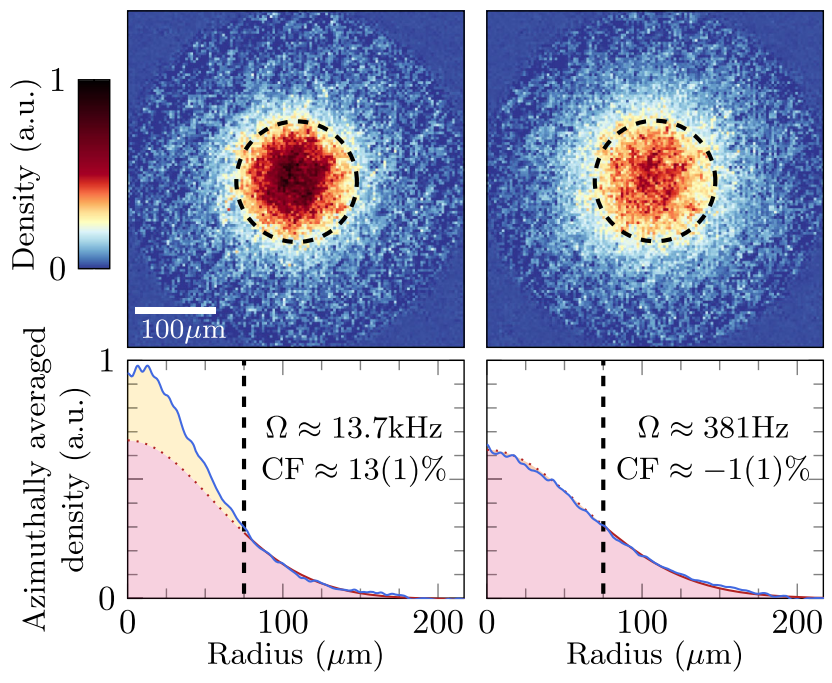

FIG. 10. Extraction of the condensate fraction. Upper: absorption images of high (left) and low (right) condensate fraction averaged over ten experimental repetitions. Lower: the corresponding azimuthally averaged signals (blue lines). We fit Eq. (B1) (red line) to the thermal wings at radii $>R_{\text {mask }}$ (dashed black line). The condensate fraction is defined by the integrated signal above the fit extrapolation (yellow shading) over the total integrated signal (pink plus yellow shadings). To make a fair comparison, each of the two distributions is normalized by its total number of atoms, $61.0 \times 10^{3}$ and $68.3 \times 10^{3}$ in the right and left examples, respectively.

where the thermal de Broglie wavelength $\lambda_{T}=h /$ $\sqrt{2 \pi m k_{B} T}$ and the fugacity $z$ are fitted under the normalization constraint $N_{\text {th }}=2 \pi \int n_{\text {th }}(k) k d k$ and $N_{\text {bg }}$ accounts for the background signal. We extract the condensed population from the signal that lies above the fit (yellow shading in Fig. 10). The mask radius $R_{\text {mask }}$ should be large enough to leave only the thermal wings for fitting. When we analyze data taken with no condensate, we observe that the width of the fitted distribution is almost independent of the mask radius up to around $R_{\text {mask }} \approx 80 \mu \mathrm{m}$. For larger radii, the signal in the remaining thermal wings is too weak, and the fit exhibits a systematic deviation. Therefore, we set the mask radius to $R_{\text {mask }}=75 \mu \mathrm{m}$. Finally, we note that the CF values we obtain are close to those reported in Ref. [80], taking into account our measured reduced temperature.

[1] I. Bloch, J. Dalibard, and W. Zwerger, Many-Body Physics with Ultracold Gases, Rev. Mod. Phys. 80, 885 (2008).

[2] N. Goldman and J. Dalibard, Periodically Driven Quantum Systems: Effective Hamiltonians and Engineered Gauge Fields, Phys. Rev. X 4, 031027 (2014).

[3] M. Bukov, L. D’Alessio, and A. Polkovnikov, Universal High-Frequency Behavior of Periodically Driven Systems: From Dynamical Stabilization to Floquet Engineering, Adv. Phys. 64, 139 (2015). 
[4] A. Eckardt, Colloquium: Atomic Quantum Gases in Periodically Driven Optical Lattices, Rev. Mod. Phys. 89, 011004 (2017).

[5] F. Grossmann, T. Dittrich, P. Jung, and P. Hanggi, Coherent Destruction of Tunneling, Phys. Rev. Lett. 67, 516 (1991).

[6] M. Grifoni and P. Hanggi, Driven Quantum Tunneling, Phys. Rep. 304, 229 (1998).

[7] H. Lignier, C. Sias, D. Ciampini, Y. Singh, A. Zenesini, O. Morsch, and E. Arimondo, Dynamical Control of MatterWave Tunneling in Periodic Potentials, Phys. Rev. Lett. 99, 220403 (2007).

[8] E. Kierig, U. Schnorrberger, A. Schietinger, J. Tomkovic, and M.K. Oberthaler, Single-Particle Tunneling in Strongly Driven Double-Well Potentials, Phys. Rev. Lett. 100, 190405 (2008).

[9] A. Zenesini, H. Lignier, D. Ciampini, O. Morsch, and E. Arimondo, Coherent Control of Dressed Matter Waves, Phys. Rev. Lett. 102, 100403 (2009).

[10] A. S. Sørensen, E. Demler, and M. D. Lukin, Fractional Quantum Hall States of Atoms in Optical Lattices, Phys. Rev. Lett. 94, 086803 (2005).

[11] M. Aidelsburger, M. Atala, M. Lohse, J. T. Barreiro, B. Paredes, and I. Bloch, Realization of the Hofstadter Hamiltonian with Ultracold Atoms in Optical Lattices, Phys. Rev. Lett. 111, 185301 (2013).

[12] H. Miyake, G. A. Siviloglou, C. J. Kennedy, W. C. Burton, and W. Ketterle, Realizing the Harper Hamiltonian with Laser-Assisted Tunneling in Optical Lattices, Phys. Rev. Lett. 111, 185302 (2013).

[13] G. Jotzu, M. Messer, R. Desbuquois, M. Lebrat, T. Uehlinger, D. Greif, and T. Esslinger, Experimental Realization of the Topological Haldane Model with Ultracold Fermions, Nature (London) 515, 237 (2014).

[14] M. S. Rudner and N. H. Lindner, Band Structure Engineering and Non-equilibrium Dynamics in Floquet Topological Insulators, Nat. Rev. Phys. 2, 229 (2020).

[15] K. Wintersperger, C. Braun, F. N. Ünal, A. Eckardt, M. D. Liberto, N. Goldman, I. Bloch, and M. Aidelsburger, Realization of an Anomalous Floquet Topological System with Ultracold Atoms, Nat. Phys. 16, 1058 (2020).

[16] A. Lazarides, A. Das, and R. Moessner, Equilibrium States of Generic Quantum Systems Subject to Periodic Driving, Phys. Rev. E 90, 012110 (2014).

[17] L. D'Alessio and M. Rigol, Long-Time Behavior of Isolated Periodically Driven Interacting Lattice Systems, Phys. Rev. X 4, 041048 (2014).

[18] P. Ponte, A. Chandran, Z. Papić, and D. A. Abanin, Periodically Driven Ergodic and Many-Body Localized Quantum Systems, Ann. Phys. (Amsterdam) 353, 196 (2015).

[19] T. Mori, T. Kuwahara, and K. Saito, Rigorous Bound on Energy Absorption and Generic Relaxation in Periodically Driven Quantum Systems, Phys. Rev. Lett. 116, 120401 (2016).

[20] T. Kuwahara, T. Mori, and K. Saito, Floquet-Magnus Theory and Generic Transient Dynamics in Periodically Driven Many-Body Quantum Systems, Ann. Phys. (Amsterdam) 367, 96 (2016).
[21] D. A. Abanin, W. De Roeck, W. W. Ho, and F. Huveneers, Effective Hamiltonians, Prethermalization, and Slow Energy Absorption in Periodically Driven Many-Body Systems, Phys. Rev. B 95, 014112 (2017).

[22] D. V. Else, B. Bauer, and C. Nayak, Prethermal Phases of Matter Protected by Time-Translation Symmetry, Phys. Rev. X 7, 011026 (2017).

[23] F. Machado, G. D. Kahanamoku-Meyer, D. V. Else, C. Nayak, and N. Y. Yao, Exponentially Slow Heating in Short and Long-Range Interacting Floquet Systems, Phys. Rev. Research 1, 033202 (2019).

[24] K. Agarwal and I. Martin, Dynamical Enhancement of Symmetries in Many-Body Systems, Phys. Rev. Lett. 125, 080602 (2020).

[25] A. Rubio-Abadal, M. Ippoliti, S. Hollerith, D. Wei, J. Rui, S. L. Sondhi, V. Khemani, C. Gross, and I. Bloch, Floquet Prethermalization in a Bose-Hubbard System, Phys. Rev. X 10, 021044 (2020).

[26] The BCS-BEC Crossover and the Unitary Fermi Gas, edited by W. Zwerger (Springer, Berlin, 2012).

[27] A. Schirotzek, Y. I. Shin, C. H. Schunck, and W. Ketterle, Determination of the Superfluid Gap in Atomic Fermi Gases by Quasiparticle Spectroscopy, Phys. Rev. Lett. 101, 140403 (2008).

[28] S. Nascimbène, N. Navon, K. J. Jiang, F. Chevy, and C. Salomon, Exploring the Thermodynamics of a Universal Fermi Gas, Nature (London) 463, 1057 (2010).

[29] M. Horikoshi, S. Nakajima, M. Ueda, and T. Mukaiyama, Measurement of Universal Thermodynamic Functions for a Unitary Fermi Gas, Science 327, 442 (2010).

[30] M. J. H. Ku, A. T. Sommer, L. W. Cheuk, and M. W. Zwierlein, Revealing the Superfluid Lambda Transition in the Universal Thermodynamics of a Unitary Fermi Gas, Science 335, 563 (2012).

[31] D. E. Miller, J. K. Chin, C. A. Stan, Y. Liu, W. Setiawan, C. Sanner, and W. Ketterle, Critical Velocity for Superfluid Flow across the BEC-BCS Crossover, Phys. Rev. Lett. 99, 070402 (2007).

[32] T. E. Drake, Y. Sagi, R. Paudel, J. T. Stewart, J. P. Gaebler, and D. S. Jin, Direct Observation of the Fermi Surface in an Ultracold Atomic Gas, Phys. Rev. A 86, 031601(R) (2012).

[33] Y. Sagi, T. E. Drake, R. Paudel, and D. S. Jin, Measurement of the Homogeneous Contact of a Unitary Fermi Gas, Phys. Rev. Lett. 109, 220402 (2012).

[34] Y. Sagi, T. E. Drake, R. Paudel, R. Chapurin, and D. S. Jin, Probing Local Quantities in a Strongly Interacting Fermi Gas, J. Phys. Conf. Ser. 467, 012010 (2013).

[35] Y. Sagi, T. E. Drake, R. Paudel, R. Chapurin, and D. S. Jin, Breakdown of the Fermi Liquid Description for Strongly Interacting Fermions, Phys. Rev. Lett. 114, 075301 (2015).

[36] C. Carcy, S. Hoinka, M. G. Lingham, P. Dyke, C. C. N. Kuhn, H. Hu, and C. J. Vale, Contact and Sum Rules in a Near-Uniform Fermi Gas at Unitarity, Phys. Rev. Lett. 122, 203401 (2019).

[37] Y. Shin, M. W. Zwierlein, C. H. Schunck, A. Schirotzek, and W. Ketterle, Observation of Phase Separation in a Strongly Interacting Imbalanced Fermi Gas, Phys. Rev. Lett. 97, 030401 (2006). 
[38] A. L. Gaunt, T. F. Schmidutz, I. Gotlibovych, R. P. Smith, and Z. Hadzibabic, Bose-Einstein Condensation of Atoms in a Uniform Potential, Phys. Rev. Lett. 110, 200406 (2013).

[39] L. Chomaz, L. Corman, T. Bienaimé, R. Desbuquois, C. Weitenberg, S. Nascimbène, J. Beugnon, and J. Dalibard, Emergence of Coherence via Transverse Condensation in a Uniform Quasi-Two-Dimensional Bose Gas, Nat. Commun. 6, 6162 (2015).

[40] B. Mukherjee, Z. Yan, P. B. Patel, Z. Hadzibabic, T. Yefsah, J. Struck, and M. W. Zwierlein, Homogeneous Atomic Fermi Gases, Phys. Rev. Lett. 118, 123401 (2017).

[41] K. Hueck, N. Luick, L. Sobirey, J. Siegl, T. Lompe, and H. Moritz, Two-Dimensional Homogeneous Fermi Gases, Phys. Rev. Lett. 120, 060402 (2018).

[42] K. Shibata, H. Ikeda, R. Suzuki, and T. Hirano, Compensation of Gravity on Cold Atoms by a Linear Optical Potential, Phys. Rev. Research 2, 013068 (2020).

[43] S. Giorgini, L. P. Pitaevskii, and S. Stringari, Theory of Ultracold Atomic Fermi Gases, Rev. Mod. Phys. 80, 1215 (2008).

[44] P. Torma, in Cold Atoms (Imperial College, London, 2014), pp. 199-250.

[45] M. W. Zwierlein, Z. Hadzibabic, S. Gupta, and W. Ketterle, Spectroscopic Insensitivity to Cold Collisions in a TwoState Mixture of Fermions, Phys. Rev. Lett. 91, 250404 (2003).

[46] J.-R. Li, B. Shteynas, and W. Ketterle, Floquet Heating in Interacting Atomic Gases with an Oscillating Force, Phys. Rev. A 100, 033406 (2019).

[47] J. L. Ville, T. Bienaimé, R. Saint-Jalm, L. Corman, M. Aidelsburger, L. Chomaz, K. Kleinlein, D. Perconte, S. Nascimbène, J. Dalibard, and J. Beugnon, Loading and Compression of a Single Two-Dimensional Bose Gas in an Optical Accordion, Phys. Rev. A 95, 013632 (2017).

[48] K. Hueck, A. Mazurenko, N. Luick, T. Lompe, and H. Moritz, Note: Suppression of kHz-Frequency Switching Noise in Digital Micro-mirror Devices, Rev. Sci. Instrum. 88, 016103 (2017).

[49] C. Shkedrov, Y. Florshaim, G. Ness, A. Gandman, and Y. Sagi, High-Sensitivity rf Spectroscopy of a Strongly Interacting Fermi Gas, Phys. Rev. Lett. 121, 093402 (2018).

[50] S. Gupta, Radio-Frequency Spectroscopy of Ultracold Fermions, Science 300, 1723 (2003).

[51] S. Tung, G. Lamporesi, D. Lobser, L. Xia, and E. A. Cornell, Observation of the Presuperfluid Regime in a Two-Dimensional Bose Gas, Phys. Rev. Lett. 105, 230408 (2010).

[52] P. A. Murthy, D. Kedar, T. Lompe, M. Neidig, M. G. Ries, A. N. Wenz, G. Zürn, and S. Jochim, Matter-Wave Fourier Optics with a Strongly Interacting Two-Dimensional Fermi Gas, Phys. Rev. A 90, 043611 (2014).

[53] C. Shkedrov, G. Ness, Y. Florshaim, and Y. Sagi, In Situ Momentum-Distribution Measurement of a Quantum Degenerate Fermi Gas Using Raman Spectroscopy, Phys. Rev. A 101, 013609 (2020).

[54] G. Ness, C. Shkedrov, Y. Florshaim, O. K. Diessel, J. von Milczewski, R. Schmidt, and Y. Sagi, Observation of a
Smooth Polaron-Molecule Transition in a Degenerate Fermi Gas, Phys. Rev. X 10, 041019 (2020).

[55] C. Shkedrov, High-Sensitivity rf and Raman Spectroscopy of a Quantum Degenerate Fermi Gas, Ph.D. thesis, Technion-Israel Institute of Technology, 2020.

[56] A similar approach was recently reported by Martin Schlederer, A. Mozdzen, T. Lompe, and H. Moritz, Single-Atom Counting in a Two-Color Magneto-Optical Trap, Phys. Rev. A 103, 033308 (2021).

[57] R. B. Blackman, The Measurement of Power Spectra from the Point of View of Communications Engineering (Dover, New York, 1959).

[58] M. Greiner, C. A. Regal, and D. S. Jin, Emergence of a Molecular Bose-Einstein Condensate from a Fermi Gas, Nature (London) 426, 537 (2003).

[59] C. A. Regal, M. Greiner, and D.S. Jin, Observation of Resonance Condensation of Fermionic Atom Pairs, Phys. Rev. Lett. 92, 040403 (2004).

[60] M. W. Zwierlein, J. R. Abo-Shaeer, A. Schirotzek, C. H. Schunck, and W. Ketterle, Vortices and Superfluidity in a Strongly Interacting Fermi Gas, Nature (London) 435, 1047 (2005).

[61] M. W. Zwierlein, C. A. Stan, C. H. Schunck, S. M. F. Raupach, A. J. Kerman, and W. Ketterle, Condensation of Pairs of Fermionic Atoms near a Feshbach Resonance, Phys. Rev. Lett. 92, 120403 (2004).

[62] J. L. Roberts, N. R. Claussen, S. L. Cornish, and C. E. Wieman, Magnetic Field Dependence of Ultracold Inelastic Collisions near a Feshbach Resonance, Phys. Rev. Lett. 85, 728 (2000).

[63] C. A. Regal, C. Ticknor, J. L. Bohn, and D. S. Jin, Tuning p-Wave Interactions in an Ultracold Fermi Gas of Atoms, Phys. Rev. Lett. 90, 053201 (2003).

[64] X. Du, Y. Zhang, and J. E. Thomas, Inelastic Collisions of a Fermi Gas in the BEC-BCS Crossover, Phys. Rev. Lett. 102, 250402 (2009).

[65] S. Tan, Energetics of a Strongly Correlated Fermi Gas, Ann. Phys. (Amsterdam) 323, 2952 (2008).

[66] S. Tan, Generalized Virial Theorem and Pressure Relation for a Strongly Correlated Fermi Gas, Ann. Phys. (Amsterdam) 323, 2987 (2008).

[67] S. Tan, Large Momentum Part of a Strongly Correlated Fermi Gas, Ann. Phys. (Amsterdam) 323, 2971 (2008).

[68] E. Braaten and L. Platter, Exact Relations for a StronglyInteracting Fermi Gas from the Operator Product Expansion, Phys. Rev. Lett. 100, 205301 (2008).

[69] S. Zhang and A. J. Leggett, Universal Properties of the Ultracold Fermi Gas, Phys. Rev. A 79, 023601 (2009).

[70] J. T. Stewart, J. P. Gaebler, T. E. Drake, and D. S. Jin, Verification of Universal Relations in a Strongly Interacting Fermi Gas, Phys. Rev. Lett. 104, 235301 (2010).

[71] E. D. Kuhnle, H. Hu, X.-J. Liu, P. Dyke, M. Mark, P. D. Drummond, P. Hannaford, and C. J. Vale, Universal Behavior of Pair Correlations in a Strongly Interacting Fermi Gas, Phys. Rev. Lett. 105, 070402 (2010).

[72] G. B. Partridge, K. E. Strecker, R. I. Kamar, M. W. Jack, and R. G. Hulet, Molecular Probe of Pairing in the BECBCS Crossover, Phys. Rev. Lett. 95, 020404 (2005). 
[73] F. Werner, L. Tarruell, and Y. Castin, Number of Closed-Channel Molecules in the BEC-BCS Crossover, Eur. Phys. J. B 68, 401 (2009).

[74] E. D. Kuhnle, S. Hoinka, P. Dyke, H. Hu, P. Hannaford, and C. J. Vale, Temperature Dependence of the Universal Contact Parameter in a Unitary Fermi Gas, Phys. Rev. Lett. 106, 170402 (2011).

[75] M. G. Lingham, K. Fenech, T. Peppler, S. Hoinka, P. Dyke, P. Hannaford, and C. J. Vale, Bragg Spectroscopy of Strongly Interacting Fermi Gases, J. Mod. Opt. 63, 1783 (2016).

[76] N. Navon, S. Nascimbène, F. Chevy, and C. Salomon, The Equation of State of a Low-Temperature Fermi Gas with Tunable Interactions, Science 328, 729 (2010).

[77] S. Hoinka, M. Lingham, K. Fenech, H. Hu, C. J. Vale, J. E. Drut, and S. Gandolfi, Precise Determination of the Structure Factor and Contact in a Unitary Fermi Gas, Phys. Rev. Lett. 110, 055305 (2013).

[78] M. Horikoshi, M. Koashi, H. Tajima, Y. Ohashi, and M. Kuwata-Gonokami, Ground-State Thermodynamic Quantities of Homogeneous Spin-1/2 Fermions from the BCS Region to the Unitarity Limit, Phys. Rev. X 7, 041004 (2017).

[79] S. Laurent, M. Pierce, M. Delehaye, T. Yefsah, F. Chevy, and C. Salomon, Connecting Few-Body Inelastic Decay to Quantum Correlations in a Many-Body System: A Weakly Coupled Impurity in a Resonant Fermi Gas, Phys. Rev. Lett. 118, 103403 (2017).

[80] B. Mukherjee, P. B. Patel, Z. Yan, R. J. Fletcher, J. Struck, and M. W. Zwierlein, Spectral Response and Contact of the Unitary Fermi Gas, Phys. Rev. Lett. 122, 203402 (2019).

[81] F. Palestini, A. Perali, P. Pieri, and G. C. Strinati, Temperature and Coupling Dependence of the Universal Contact Intensity for an Ultracold Fermi Gas, Phys. Rev. A 82, 021605(R) (2010).

[82] R. Haussmann, M. Punk, and W. Zwerger, Spectral Functions and rf Response of Ultracold Fermionic Atoms, Phys. Rev. A 80, 063612 (2009).

[83] H. Hu, X. J. Liu, and P. D. Drummond, Equation of State of a Superfluid Fermi Gas in the BCS-BEC Crossover, Europhys. Lett. 74, 574 (2006).

[84] E. D. Kuhnle, H. Hu, X.-J. Liu, P. Dyke, M. Mark, P. D. Drummond, P. Hannaford, and C. J. Vale, Universal Behavior of Pair Correlations in a Strongly Interacting Fermi Gas, Phys. Rev. Lett. 105, 070402 (2010).

[85] S. Gandolfi, K. E. Schmidt, and J. Carlson, BEC-BCS Crossover and Universal Relations in Unitary Fermi Gases, Phys. Rev. A 83, 041601(R) (2011).

[86] P. Ponte, Z. Papić, F. Huveneers, and D. A. Abanin, ManyBody Localization in Periodically Driven Systems, Phys. Rev. Lett. 114, 140401 (2015).

[87] A. Lazarides, A. Das, and R. Moessner, Fate of ManyBody Localization under Periodic Driving, Phys. Rev. Lett. 115, 030402 (2015).

[88] V. Khemani, A. Lazarides, R. Moessner, and S. L. Sondhi, Phase Structure of Driven Quantum Systems, Phys. Rev. Lett. 116, 250401 (2016).

[89] P. Bordia, H. Luschen, U. Schneider, M. Knap, and I. Bloch, Periodically Driving a Many-Body Localized Quantum System, Nat. Phys. 13, 460 (2017).
[90] J. Berges, S. Borsányi, and C. Wetterich, Prethermalization, Phys. Rev. Lett. 93, 142002 (2004).

[91] M. Moeckel and S. Kehrein, Interaction Quench in the Hubbard Model, Phys. Rev. Lett. 100, 175702 (2008).

[92] M. Eckstein, M. Kollar, and P. Werner, Thermalization after an Interaction Quench in the Hubbard Model, Phys. Rev. Lett. 103, 056403 (2009).

[93] M. Moeckel and S. Kehrein, Crossover from Adiabatic to Sudden Interaction Quenches in the Hubbard Model: Prethermalization and Non-equilibrium Dynamics, New J. Phys. 12, 055016 (2010).

[94] M. Gring, M. Kuhnert, T. Langen, T. Kitagawa, B. Rauer, M. Schreitl, I. Mazets, D. A. Smith, E. Demler, and J. Schmiedmayer, Relaxation and Prethermalization in an Isolated Quantum System, Science 337, 1318 (2012).

[95] S. A. Weidinger and M. Knap, Floquet Prethermalization and Regimes of Heating in a Periodically Driven, Interacting Quantum System, Sci. Rep. 7, 45382 (2017).

[96] D. Abanin, W. D. Roeck, W. W. Ho, and F. Huveneers, A Rigorous Theory of Many-Body Prethermalization for Periodically Driven and Closed Quantum Systems, Commun. Math. Phys. 354, 809 (2017).

[97] U. Schnorrberger, J. D. Thompson, S. Trotzky, R. Pugatch, N. Davidson, S. Kuhr, and I. Bloch, Electromagnetically Induced Transparency and Light Storage in an Atomic Mott Insulator, Phys. Rev. Lett. 103, 033003 (2009).

[98] Y.-F. Hsiao, P.-J. Tsai, H.-S. Chen, S.-X. Lin, C.-C. Hung, C.-H. Lee, Y.-H. Chen, Y.-F. Chen, I. A. Yu, and Y.-C. Chen, Highly Efficient Coherent Optical Memory Based on Electromagnetically Induced Transparency, Phys. Rev. Lett. 120, 183602 (2018).

[99] Y. Wang, J. Li, S. Zhang, K. Su, Y. Zhou, K. Liao, S. Du, H. Yan, and S.-L. Zhu, Efficient Quantum Memory for Single-Photon Polarization Qubits, Nat. Photonics 13, 346 (2019).

[100] Y. Sagi, R. Pugatch, I. Almog, and N. Davidson, Spectrum of Two-Level Systems with Discrete Frequency Fluctuations, Phys. Rev. Lett. 104, 253003 (2010).

[101] Y. Sagi, I. Almog, and N. Davidson, Universal Scaling of Collisional Spectral Narrowing in an Ensemble of Cold Atoms, Phys. Rev. Lett. 105, 093001 (2010).

[102] L. Viola and S. Lloyd, Dynamical Suppression of Decoherence in Two-State Quantum Systems, Phys. Rev. A 58, 2733 (1998).

[103] L. Viola, E. Knill, and S. Lloyd, Dynamical Decoupling of Open Quantum Systems, Phys. Rev. Lett. 82, 2417 (1999).

[104] E. L. Hahn, Spin Echoes, Phys. Rev. 80, 580 (1950).

[105] Y. Sagi, I. Almog, and N. Davidson, Process Tomography of Dynamical Decoupling in a Dense Cold Atomic Ensemble, Phys. Rev. Lett. 105, 053201 (2010).

[106] H. Y. Carr and E. M. Purcell, Effects of Diffusion on Free Precession in Nuclear Magnetic Resonance Experiments, Phys. Rev. 94, 630 (1954).

[107] S. Meiboom and D. Gill, Modified Spin-Echo Method for Measuring Nuclear Relaxation Times, Rev. Sci. Instrum. 29, 688 (1958).

[108] U. Haeberlen, High Resolution NMR in Solids: Selective Averaging (Academic, New York, 1976). 
[109] S. Damodarakurup, M. Lucamarini, G. Di Giuseppe, D. Vitali, and P. Tombesi, Experimental Inhibition of Decoherence on Flying Qubits via "Bang-Bang" Control, Phys. Rev. Lett. 103, 040502 (2009).

[110] M. J. Biercuk, H. Uys, A. P. VanDevender, N. Shiga, W. M. Itano, and J. J. Bollinger, Optimized Dynamical Decoupling in a Model Quantum Memory, Nature (London) 458, 996 (2009).

[111] S. Kotler, N. Akerman, Y. Glickman, and R. Ozeri, Nonlinear Single-Spin Spectrum Analyzer, Phys. Rev. Lett. 110, 110503 (2013).

[112] J. Du, X. Rong, N. Zhao, Y. Wang, J. Yang, and R. B. Liu, Preserving Electron Spin Coherence in Solids by Optimal Dynamical Decoupling, Nature (London) 461, 1265 (2009).

[113] G. de Lange, Z. H. Wang, D. Riste, V. V. Dobrovitski, and R. Hanson, Universal Dynamical Decoupling of a Single Solid-State Spin from a Spin Bath, Science 330, 60 (2010).

[114] B. Naydenov, F. Dolde, L. T. Hall, C. Shin, H. Fedder, L. C. L. Hollenberg, F. Jelezko, and J. Wrachtrup, Dynamical Decoupling of a Single-Electron Spin at Room Temperature, Phys. Rev. B 83, 081201(R) (2011).

[115] N. Bar-Gill, L. M. Pham, A. Jarmola, D. Budker, and R. L. Walsworth, Solid-State Electronic Spin Coherence Time Approaching One Second, Nat. Commun. 4, 1743 (2013).
[116] I. Almog, Y. Sagi, G. Gordon, G. Bensky, G. Kurizki, and N. Davidson, Direct Measurement of the SystemEnvironment Coupling as a Tool for Understanding Decoherence and Dynamical Decoupling, J. Phys. B 44, 154006 (2011).

[117] D. Trypogeorgos, A. Valdés-Curiel, N. Lundblad, and I. B. Spielman, Synthetic Clock Transitions via Continuous Dynamical Decoupling, Phys. Rev. A 97, 013407 (2018).

[118] H. Edri, B. Raz, G. Fleurov, R. Ozeri, and N. Davidson, Observation of Nonlinear Spin Dynamics and Squeezing in a BEC Using Dynamic Decoupling, New J. Phys. 23, 053005 (2021).

[119] T. Kohlert, S. Scherg, P. Sala, F. Pollmann, B. H. Madhusudhana, I. Bloch, and M. Aidelsburger, Experimental Realization of Fragmented Models in Tilted FermiHubbard Chains, arXiv:2106.15586.

[120] W. Ketterle and M. W. Zwierlein, in Proceedings of the International School of Physics "Enrico Fermi," Course CLXIV, edited by M. Inguscio, W. Ketterle, and C. Salomon (IOS Press, Amsterdam, 2008).

[121] G. Ness, A. Vainbaum, C. Shkedrov, Y. Florshaim, and Y. Sagi, Single-Exposure Absorption Imaging of Ultracold Atoms Using Deep Learning, Phys. Rev. Applied 14, 014011 (2020). 